\title{
Article \\ A Numerical Study of a Compressed Air Engine with Rotating Cylinders
}

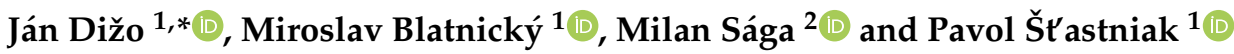 \\ 1 Department of Transport and Handling Machines, Faculty of Mechanical Engineering, University of Žilina, \\ Univerzitná 8215/1, 01026 Žilina, Slovakia; miroslav.blatnicky@fstroj.uniza.sk (M.B.); \\ pavol.stastniak@fstroj.uniza.sk (P.Š.) \\ 2 Department of Applied Mechanics, Faculty of Mechanical Engineering, University of Žilina, \\ Univerzitná 8215/1, 01026 Žilina, Slovakia; milan.saga@fstroj.uniza.sk \\ * Correspondence: jan.dizo@fstroj.uniza.sk; Tel.: +421-41-513-2560
}

Citation: Dižo, J.; Blatnický, M.; Sága, M.; Št'astniak, P. A Numerical Study of a Compressed Air Engine with Rotating Cylinders. Appl. Sci. 2021, 11, 7504. https://doi.org/ 10.3390/app11167504

Academic Editor: Ramin Rahmani

Received: 30 April 2021

Accepted: 10 August 2021

Published: 16 August 2021

Publisher's Note: MDPI stays neutral with regard to jurisdictional claims in published maps and institutional affiliations.

Copyright: (c) 2021 by the authors. Licensee MDPI, Basel, Switzerland. This article is an open access article distributed under the terms and conditions of the Creative Commons Attribution (CC BY) license (https:// creativecommons.org/licenses/by/ $4.0 /)$.

\begin{abstract}
This article explores the possibility that, during the elimination of conventional combustion engines, the connecting rod becomes deflected. A larger connecting rod angle creates higher lateral pressure on the piston, also leading to greater loads on other engine components. This fact inspired us to develop an applied mechanism design that reduces the disadvantages of conventional combustion engines. The presented mathematical model that describes the designed engine working principle was created utilizing Lagrange's equations of motion of the second kind and solved in MATLAB. This paper also includes a multibody simulation model of the engine mechanism created using the Simpack software. Based on a comparison of the two methods, the obtained waveforms of the selected kinematic quantities were found to yield minimal deviations. A real prototype was subsequently developed based on the mathematical model outputs. In this manner, we practically verified that the proposed theoretical solution for a non-conventional engine is fully functional.
\end{abstract}

Keywords: compressed air engine; rotating cylinders; non-conventional engine; numerical simulation; analysis and design

\section{Introduction}

After years of the worldwide development of internal combustion engines and their improvement, they are technical and constructional at a very high level. Therefore, it is difficult to compare them with other less conventional engines. Recently, the reduction in exhaust emissions by complete elimination of internal combustion engines is not possible. At present and in the near future, reducing exhaust emissions by completely eliminating internal combustion engines is not possible, as no technology is available to completely replace them $[1,2]$. Therefore, the issue of increasing the effectiveness of such engines needs to be addressed.

One of our goals was to present the issue of a non-conventional engine that could be used in a wide range of technical applications. To increase the use of non-conventional engines alongside dominant conventional engines, such novel engines must be more advantageous in certain areas. This condition also applies to comparisons with other pre-existing non-conventional engine design solutions. A four-stroke engine with opposed pistons, described in the patent SK 3731 Y1 [3], is known from prior studies. The disadvantage of this engine is its large dimensions and weight because this engine's connecting rods are coupled to two individual crankshafts in a sizeable planetary gear. Moreover, this engine is mounted in a reducer. Other non-conventional engine designs include a crank mechanism FIK [4,5], a wobble board [6,7], variable piston motion [8], and others [9-11].

In aircraft technology, multi-cylinder engines featuring opposed-piston movement of two pistons in one common cylinder are well known. These engines usually use a two-stroke configuration. In terms of design, these engines are characterized by having 
two crankshafts installed on both sides of the cylinder parts. A six-cylinder two-stroke straight Jumbo diesel engine is a typical example of such an engine [1,2].

The disadvantage of all piston engines is the fact that their larger connecting rod angles lead to greater lateral loading of the pistons. Similarly, other construction components are loaded and lead the lower compressive force to generate torque. Another disadvantage is that the pistons move relative to the cylinders in a rectilinear reciprocating motion. This motion places increased demands on balancing the smooth operation of engines using additional balancing components [2,12,13].

On the other hand, compressed air engines have several advantages. That essential is that they have a relatively small mass in relation to their power at relatively high permissible overloads. Further advantages are as follows: convenient was of obtaining power source, high starting torque, easy control of speed and torque, resistance to overloads, and the heat, which is generated by the friction between the internal elements and is cooled as a result of gas expansion [14-16].

Consequently, we sought to develop a kinematic mechanism configuration (Figure 1) that can reduce, as much as possible, the disadvantages of conventional piston engines. This design would be suitable primarily for piston engines and is outlined by the kinematic mechanism configuration described below. This technical solution has potential applications in the field of piston engines for both gasoline and diesel engines, in two-stroke or four-stroke engines, in the further design of compressors and hydraulic pumps, and in engines for compressed air $[17,18]$.

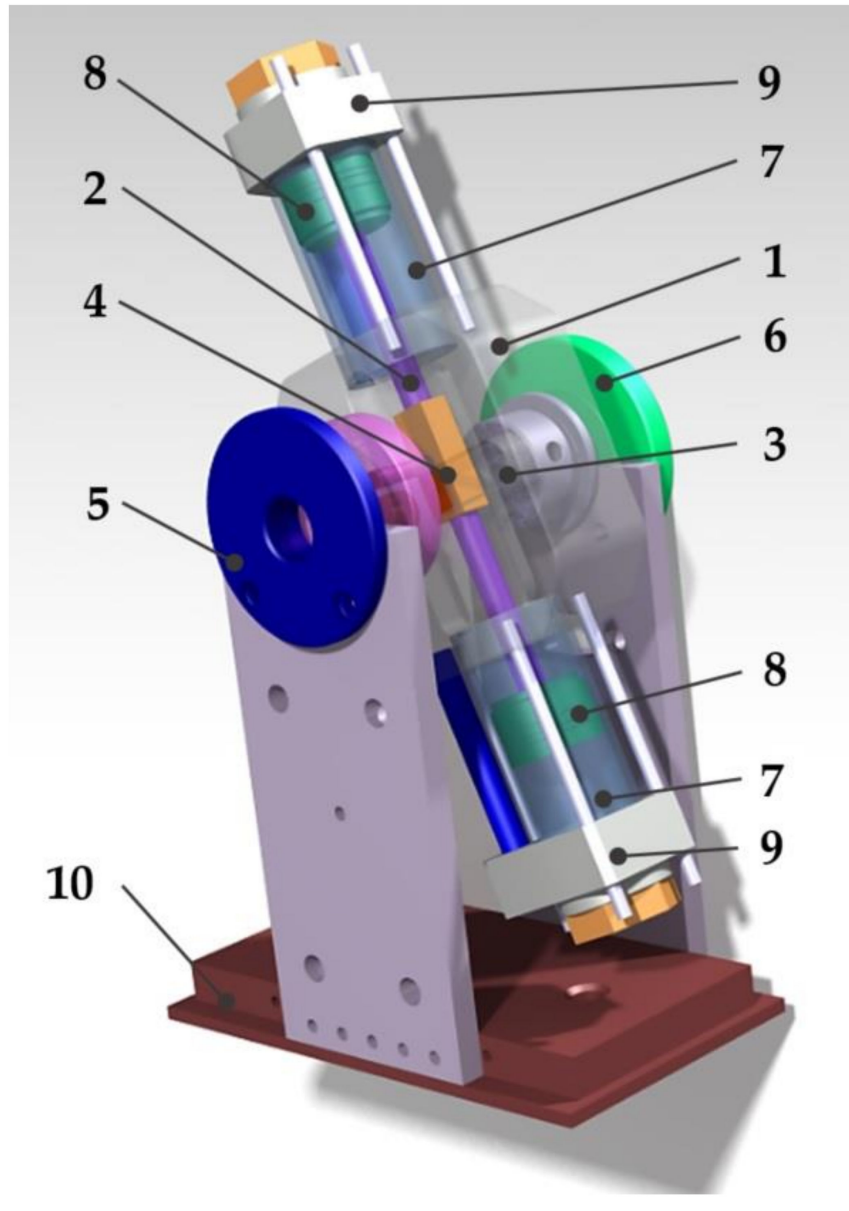

(a)

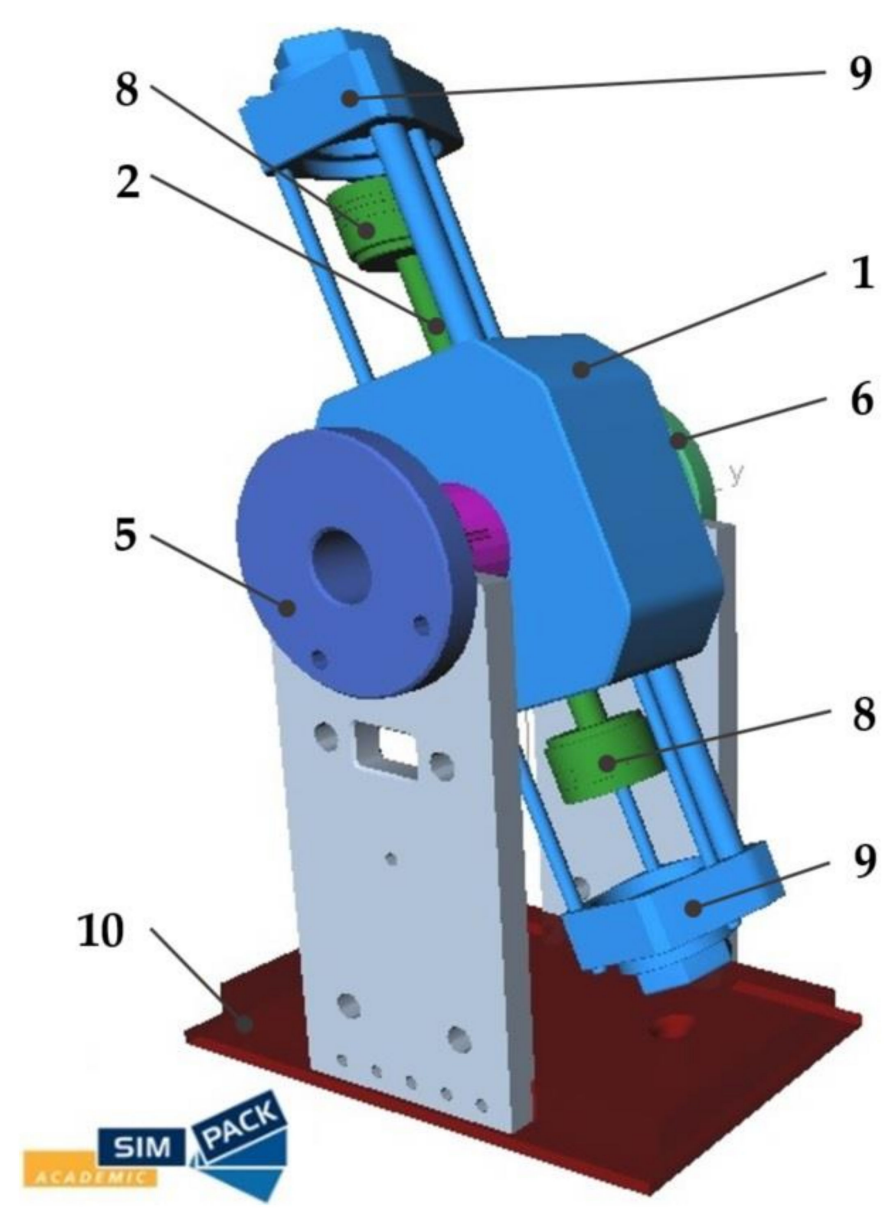

(b)

Figure 1. A non-conventional engine: (a) a three-dimensional model of a non-conventional engine design; (b) a schematic model in the Simpack software: 1-a plate, 2-a connecting rod, 3-an auxiliary stone, 4-a stone, 5-a first output, 6-a second output, 7-cylinders, 8-pistons, 9-heads of cylinders, 10-a stand. 
An expected disadvantage of the designed engine mechanism with a rotating cylinder is that relatively large masses perform a rotational movement. Although it is assumed that a couple of pistons moving against each other will eliminate imbalances as much as possible, main bearings will have to be sufficiently dimensioned. With this described characteristic, the changing mass of inertia is related. It is caused by the general planar motion of the pistons in the switchboard. The translational motion of the piston in the rotating switchboard leads so that the center of gravity of this construction unit is not still in the one point. One of the possible ways to reach a smooth angular velocity of the switchboard could be by increasing its mass, and it would also serve as a flywheel. In another way, reducing the piston mass could lead to better smoothness of the switchboard. A combination of previous methods would also be possible to use to improve the dynamic response of the mechanism during its operation. However, it is necessary and required to create and verify a computational model of the engine mechanism and to perform a numerical study of the designed engine with a rotating cylinder. Therefore, these activities are presented and described in detail in this work.

Most commonly, pneumatic mechanisms use compressed air as a working fluid. These mechanisms provide rectilinear, rotational, or oscillating motion. The use of compressed air limits the possibility of using such a mechanism. However, these mechanisms should be used under conditions that do not allow the use of any other type of mechanism for safety, hygiene, or other reasons [19]. The use of compressed engines is conditional on the compressed air; for this, a compressor serves. In pneumatic engines, an energy conversion of the compressed air to mechanical energy occurs. The advantage of the engine with a rotational motion is a relatively easy regulation of speed, the possibility to use them in any position, a minimal failure rate, and simplicity. The main disadvantage is their cost (due to the need for a compressor station and a distribution system). A pneumatic engine with a rectilinear motion is most commonly used in automatization. It is composed of the main body, a piston, and a piston rod. A pneumatic engine with an oscillating motion is, in principle, an engine with rotational motion; however, they have a limited angle of rotation. They are used in high-pressure pumps.

Therefore, there are some fields in which pneumatic mechanisms, i.e., engines, can be used. Despite a few disadvantages, some researchers and scientists developed vehicles that are powered by pneumatic engines [20-22]. The application of a drive train powered by compressed air could be more ecological because such drive trains do not employ the combustion processes of fossil fuels. Compressed air-powered propulsion systems for transport means are developed, and they attract research focuses. Alternatively, compressed air engines can be integrated as a part of hybrid systems, including a conventional combustion engine. Such a hybrid powertrain system can be considered as a transition vehicles power system between the fossil-fueled combustion engine and zero-emission propulsion system, such as an electric power unit or a compressed air powered unit [17,19,23,24].

This type of vehicle, called a pneumobil, could be used in places where the production of gas emissions is undesirable, e.g., in production halls, enclosed spaces, and town centers. Thus far, the use of compressed air engines was limited due to the insufficient supply of a medium [18].

The first pneumobil in the Czech Republic was designed at the Brno University of Technology in 2011 [25]. This vehicle is powered by compressed air stored in air reservoirs. The very first prototype ran a track with a length of $3 \mathrm{~km}$ at a speed of approximately $40 \mathrm{~km} / \mathrm{h}$. In 2018, this pneumobil was able to run a $7 \mathrm{~km}$ track at a speed of $50 \mathrm{~km} / \mathrm{h}$ [26].

In Slovakia, researchers from the Technical University of Košice introduced their first pneumobil in 2018 [27]. This vehicle is equipped with a three-cylinder engine powered by compressed air with a displacement of 1.91 . The air reservoir has a volume of $10 \mathrm{~L}$, and the maximal pressure of the compressed air is $20 \mathrm{MPa}$.

Research on the application of compressed air engines is also being performed at other institutions. The majority of prototypes of the compressed air engines come from modifications of conventional internal combustion engines. For instance, researchers from 
Zhejiang University conducted studies with a modified single-cylinder diesel engine, and they created an engine from compressed air. Its maximal power reached the value of $2.6 \mathrm{~kW}$ at an air pressure of $0.8 \mathrm{MPa}[19,28]$.

The team from the National Tsing Hua University developed compressed air engines based on a conventional motorcycle engine. The experiments proved the maximal power of $0.96 \mathrm{~kW}$ at the air pressure of $0.9 \mathrm{MPa}$. A modified engine has had the maximal power of $2.15 \mathrm{~kW}$ under the air pressure of $1.3 \mathrm{MPa}$, and the motorcycle was able to drive at the speed of $38.2 \mathrm{~km} / \mathrm{h}$ for $5 \mathrm{~km}[19,21]$.

Although the pneumobil is currently used in the automotive industry's development and research competitions between universities [29,30], it is still being developed and improved. The main goal is to develop an easily handled vehicle that would contribute to reducing environmental impacts and noise while driving as long a distance as possible [23]. Moreover, the automotive company PSA worked on the development of a car powered by compressed air that would replace the electromotors in the company's hybrid vehicles [31].

Tata Motors of India was another vehicle manufacturer that developed a vehicle powered by a pneumatic engine. The compressed air engine should be used in the modified model Nano, which was previously under consideration as part of their collaboration with the MDI vehicle manufacturer [19,32].

There were also other lesser-known companies that have worked on the development of a compressed air engine. The air engine Di Pietro motor is a product of the EngineAir of Australia Company. It is a simple cylindrical rotary piston engine, and this piston moves without any friction inside the cylindrical stator [19,33].

The idea of hybridization, including the pneumatic engines mentioned above, is alive thanks to the Scania company. It consists of decreasing fuel consumption by using the braking energy, which may be lost. Heavy Scania engines, which were converted to operate as pneumatic engines, operate during the braking of a vehicle, and during its acceleration, the engine may work as a pneumatic engine powered by the previously accumulated pressurized air. As simulations have shown, a conventional bus may have decreased fuel consumption by as much as $58 \%$ after converting it into a pneumatic hybrid bus $[14,34]$.

\section{Materials and Methods}

For reasons of cleanliness, we chose a practical design of a mechanism in which compressed air energy is changed into mechanical energy in the form of the cylinder's translational movement. Subsequently, the crank mechanism changes the energy into the rotating movement of the shafts. The working principle of our construction design is provided in Figure 2.

The design consists of a mechanism formed by a plate (1) and an extra-level configuration of a connecting rod (2) along with an auxiliary groove (3). An axis rotation (4) of the entire link mechanism is located at the extra-level intersection. A shaft (5) is also placed in the axis rotation (4). In the axis rotation, two cylinders (5) are mounted opposite to each other and radially relative to the link mechanism. A cylinder head (6) sits above the cylinders. As usual, the pistons (7) are located in the cylinders and interlinked by a mutual connecting rod (8). The connecting rod then passes through the connecting rod groove (2). At the same, the connecting rod stone (9) is placed in the middle of the connecting rod. The auxiliary stone (10) is located in the auxiliary groove (3). One pin (11) of the crankshaft matches the connecting rod stone slot, and the other pin (12) of the crankshaft matches the auxiliary stone slot. The axis rotation of the crankshaft is located between these two pins of the crankshaft. The axis rotation of the link mechanism and the axis rotation of the crankshaft are abaxial. The crankshaft radius equals $\frac{1}{4}$ of the piston stroke length. The axis rotation of the link mechanism is the output of the mechanism with revolutions $n_{1}$. The axis rotation of the crankshaft is the output with revolutions $n_{2}$, where $n_{2}=2 \cdot n_{1}$. 


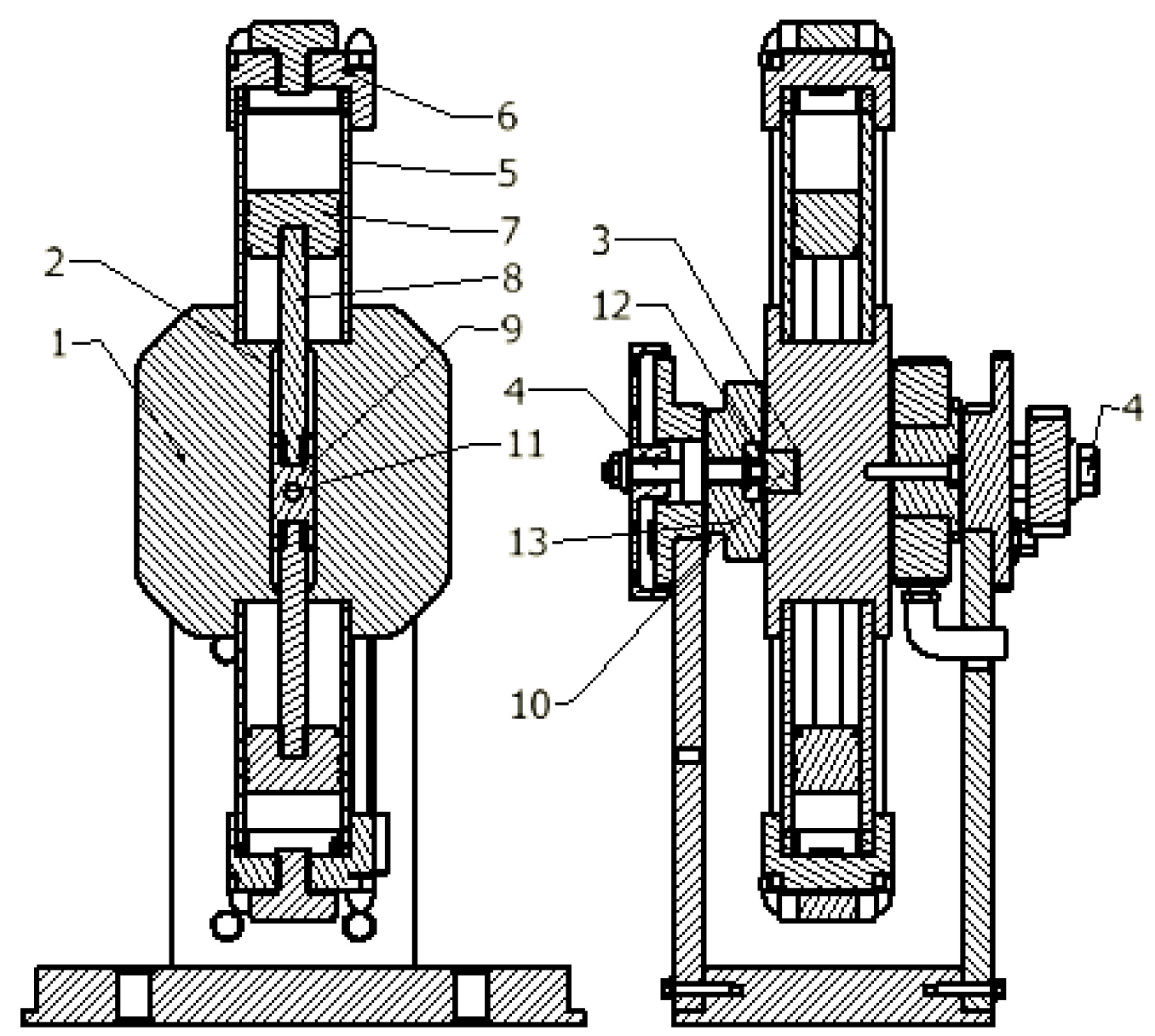

Figure 2. A cross section of the designed mechanism to illustrate the component configuration.

A plate was adapted to distribute the working medium. The distribution of the working medium is accomplished by means of a crosshead that includes the extra-level grooves (2) and (3). The input of the working medium is placed on the opposite cylinder side and inserted using a metal tube. The output from the functional block is located on both sides. One side includes only the shaft output, which is mounted to a stand. The other side is connected to a gearbox with a gear ratio of $i^{g}=0.5$. The shaft output from the gearbox leads to an attachment of the engine stand. Detailed drawings of the selected main bodies are shown in Figures 3-6.

The exact cylinder head geometry was used to build a real engine prototype to verify the engine's functionality.

The advantages of the designed mechanism are obvious from its external effects. These effects include total elimination of the lateral (normal) force on the piston. This occurs due to the connecting rod's angle of zero degrees. The connecting rod moves only in the cylinder axis. Furthermore, the rotating cylinder blocks and cylinders act as a flywheel, significantly reducing the weight of the engine. We assume that, for certain applications, a liquid cooling system is not needed because the rotating cylinders dissipate heat into the ambient air in which the cylinders move. This ensures sufficient circulation of the air, and the Grossman factor is increased for heat removal from the rotating cylinders. Lastly, the torque output from the rotating cylinders is reached without toothed gears. This results in a reduction in the lubrication requirements of the mechanism and increases the efficiency of the mechanism due to the reduced friction. The presented design was granted protection by the Industrial Property Office of the Slovak Republic under number SK 8035 Y1 [35]. 

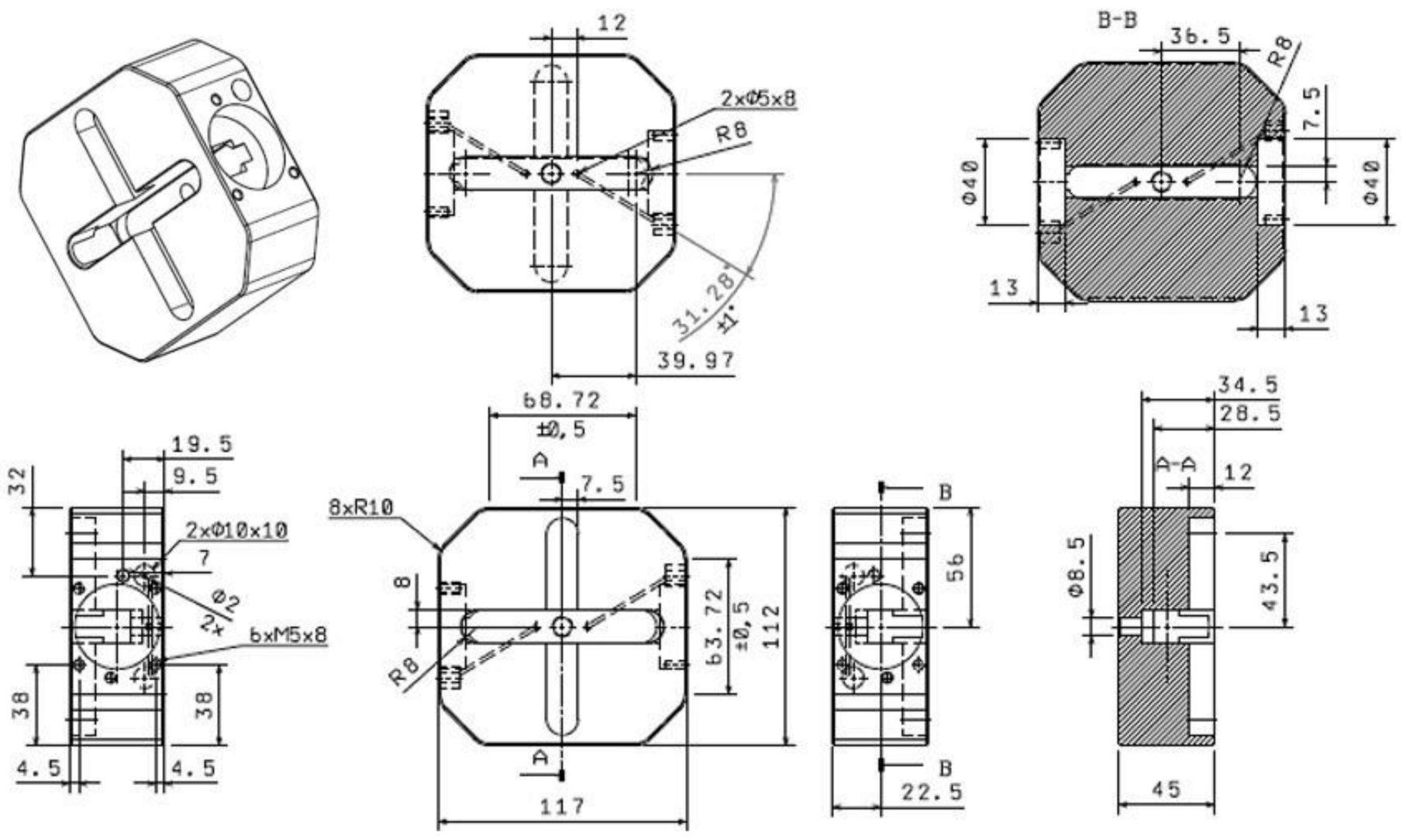

Figure 3. Exact board geometry used to build a real engine prototype to verify its functionality.
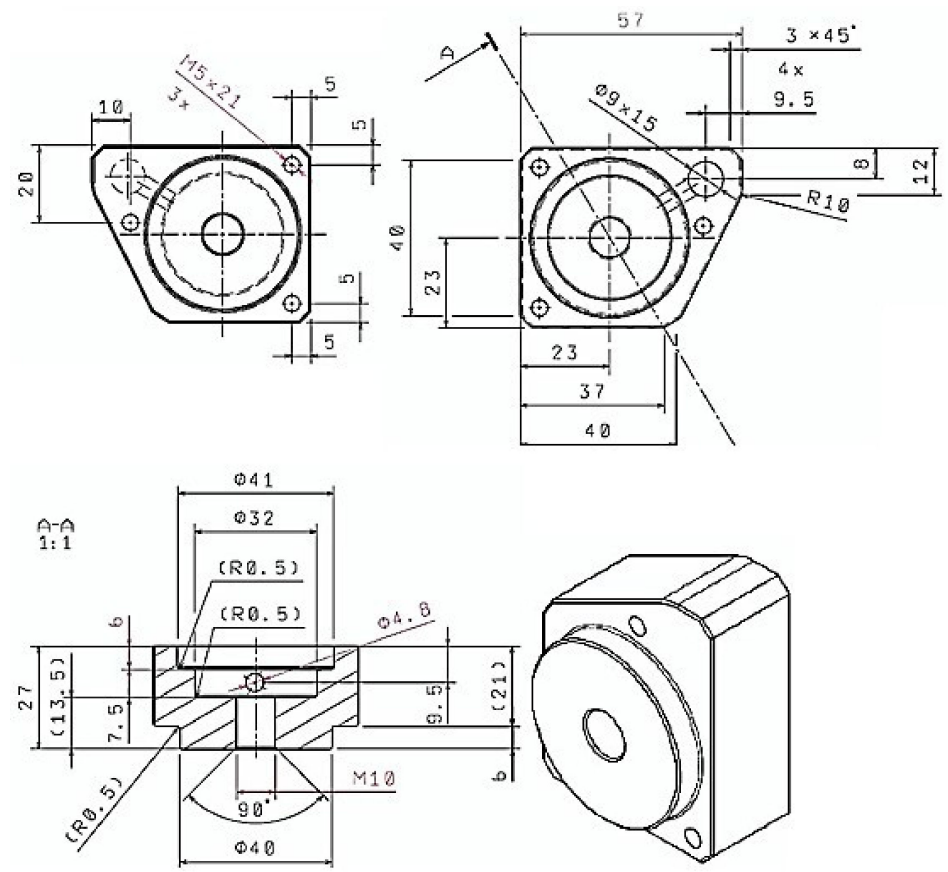

Figure 4. The exact cylinder head geometry used to build a real engine prototype to verify its functionality. 


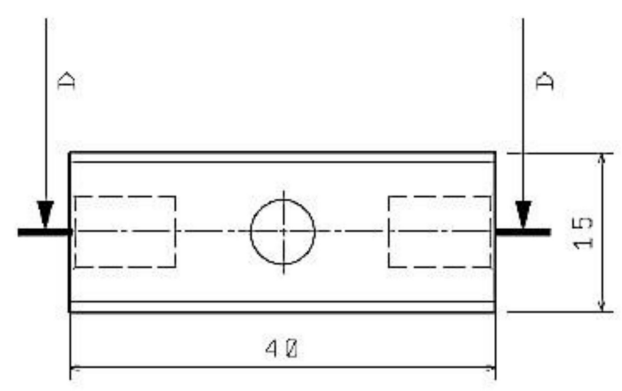

$A^{-} \hat{A}(2: 1)$

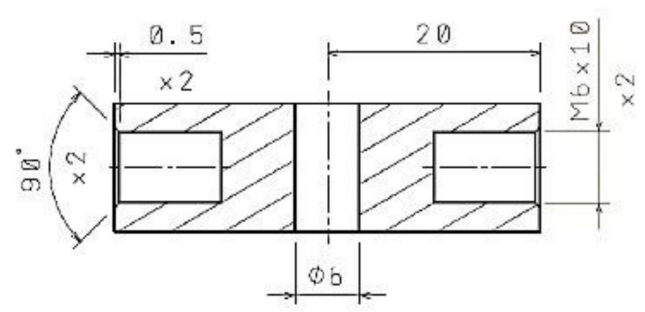

(a)
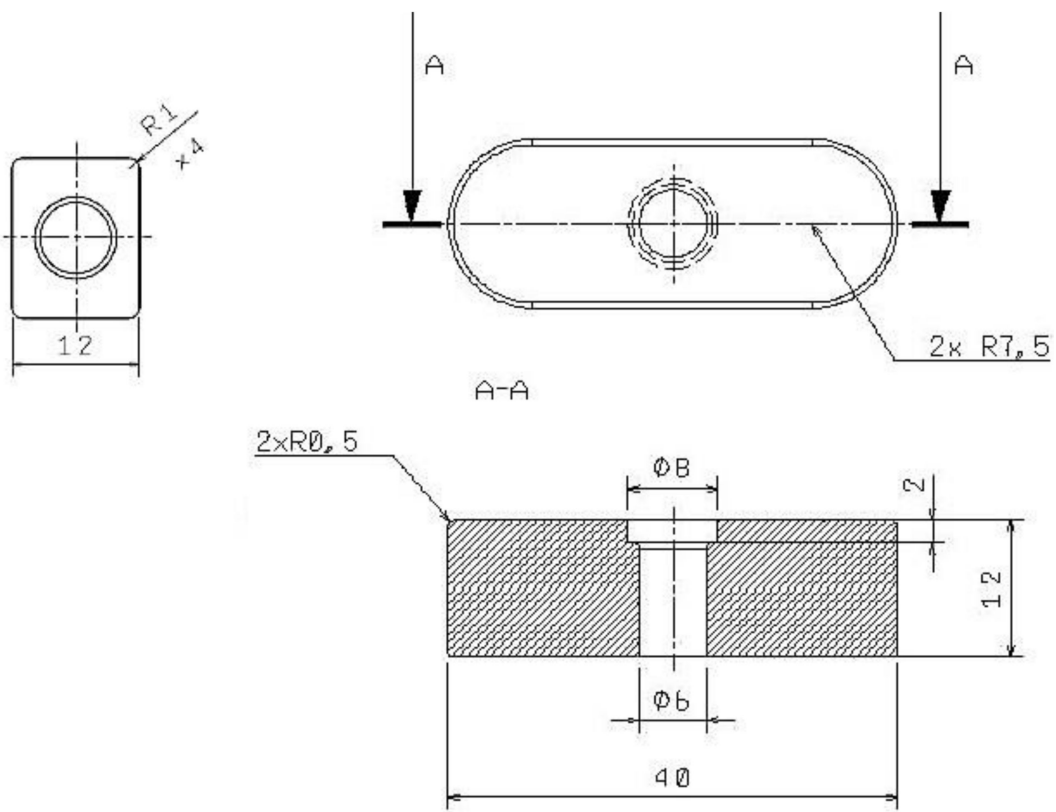

(b)

Figure 5. The exact geometry of the connecting rod stone (a) and auxiliary stone (b) used to build a real engine prototype to verify its functionality.
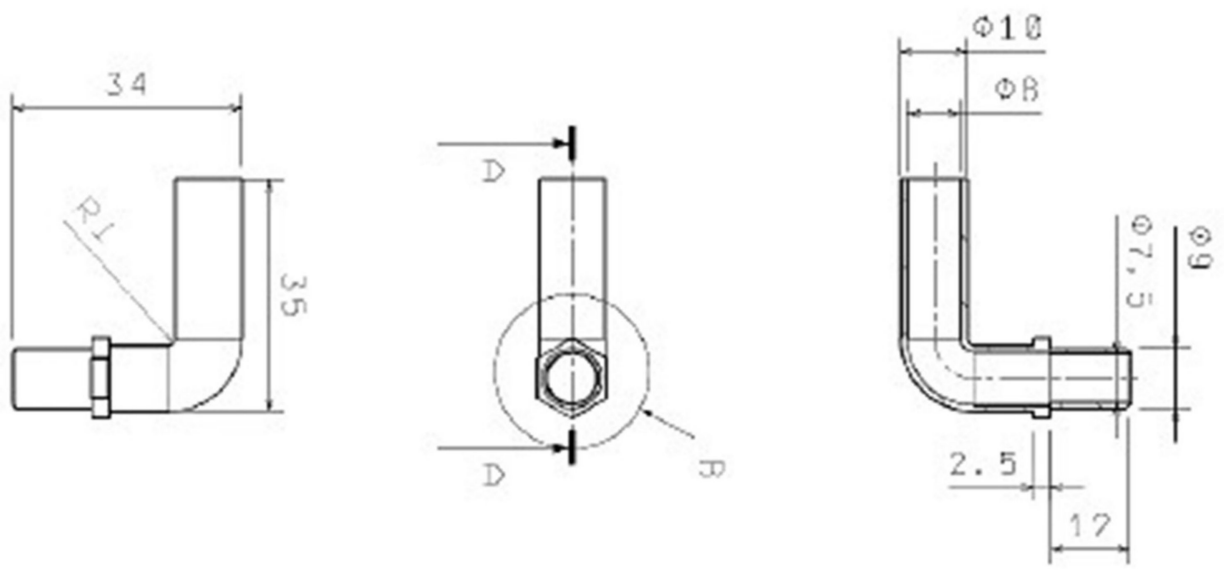

Figure 6. The exact geometry of the tube prototype used for the entry of the working medium into the cylinder space.

\section{Derivation of a Mathematical Model}

The production of a safe physical prototype is quite complicated. Prototyping must be preceded by a great deal of scientific research (i.e., simulation computations) and manual work to meet the most important requirement: safety.

The equation of motion describing the dynamic behavior of the mechanism can be derived by means of Lagrange's equations of motion of the second kind, which come from the principle of virtual work in the following form [36-38]:

$$
\frac{d}{d t}\left(\frac{\partial E_{K}}{\partial \dot{q}_{i}}\right)-\frac{\partial E_{K}}{\partial q_{i}}+\frac{\partial E_{D}}{\partial \dot{q}_{i}}+\frac{\partial E_{P}}{\partial q_{i}}=Q_{i}, i=1,2, \ldots n,
$$

where $E_{K}$ is the kinetic energy; $E_{D}$ is the dissipative energy; $E_{P}$ is the potential energy; $q$ and $\dot{q}$ are generalized coordinates and their time derivation (i.e., velocity), respectively; $i$ is the number of degrees of freedom; and $Q$ is the sum of external acting loads (forces from other types of mechanism excitation). 
To derive the mathematical model given by the equation of motion, we used the scheme shown in Figure 7.
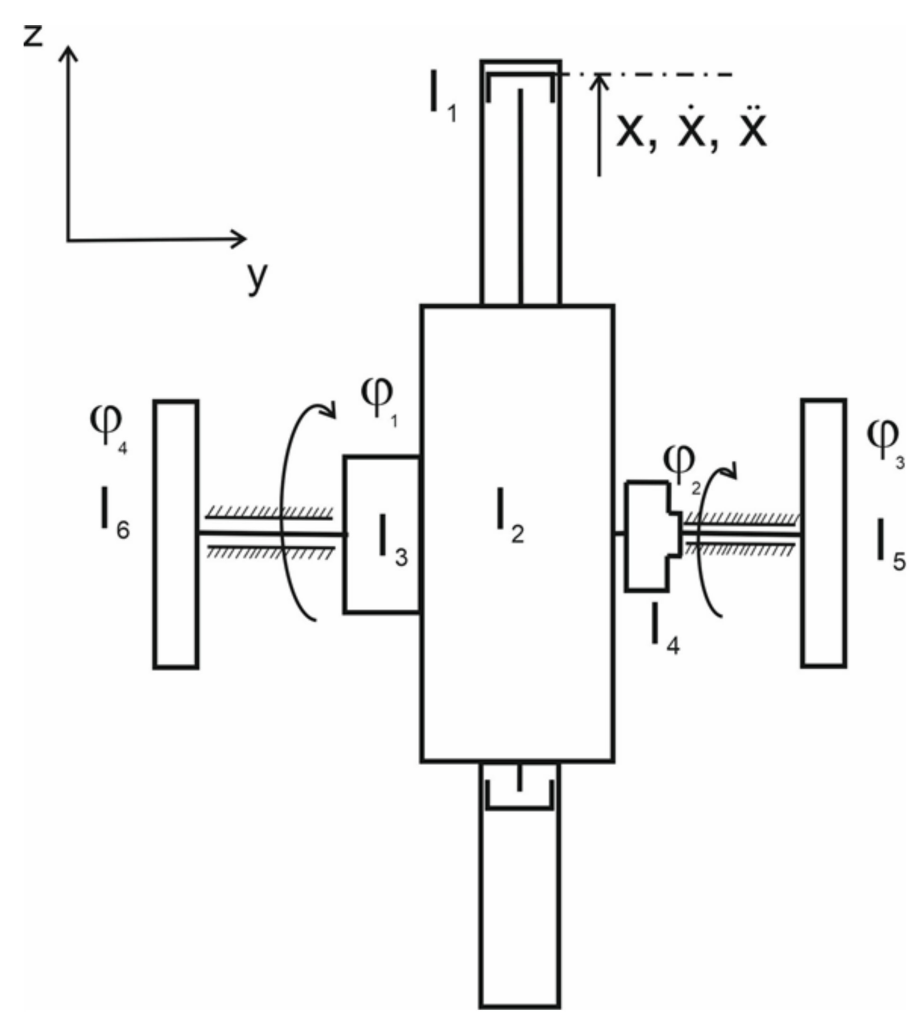

(a)
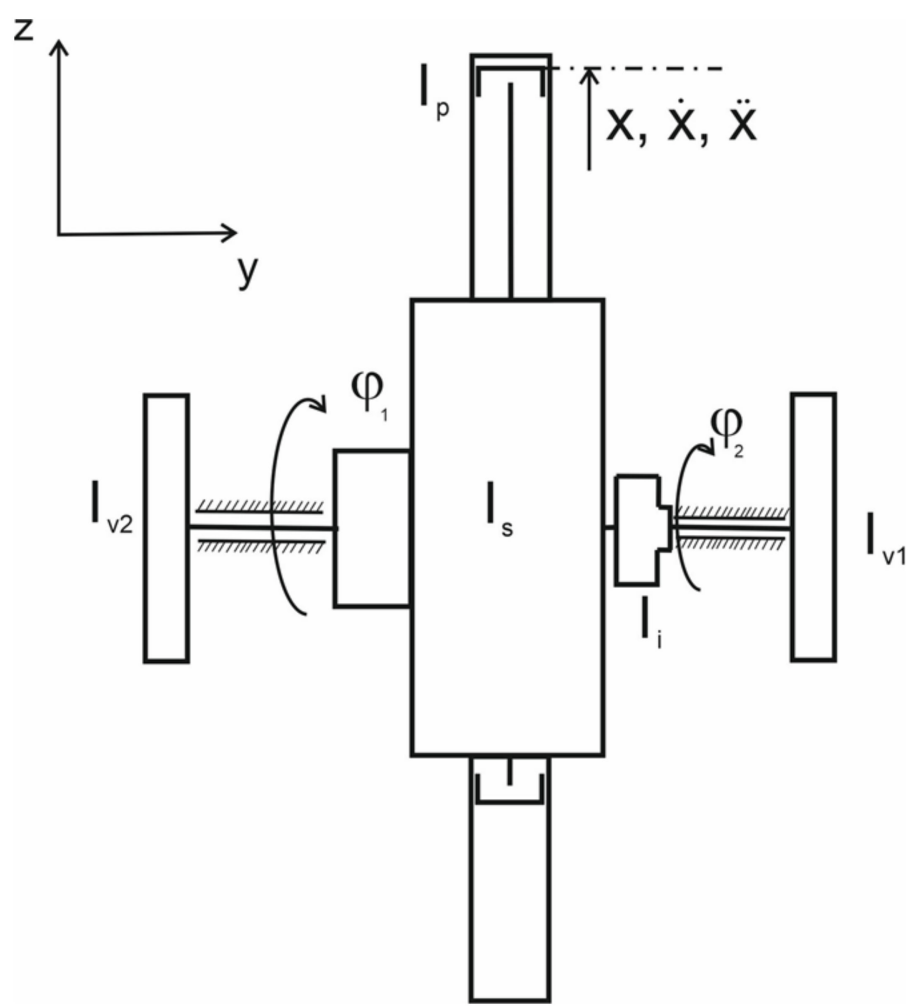

(b)

Figure 7. The calculation scheme of the mechanism: (a) a plane scheme of the designed mechanism with all components depicted; (b) a plane scheme of the designed mechanism with the components reduced to rotating components.

As the entire mechanism has one degree of freedom, we obtain one equation of motion. Firstly, we have to prescribe considered generalized coordinates. The coordinates of the rotating components are denoted as $\varphi_{i}$, where the upper index $i$ indicates individual components (Figure 7). Bodies acting with the translational movement have a generalized coordinate $x$. As only one body moves translationally, only one coordinate $x$ is used (Figure 7).

Next, we proceed to determine the individual energies. We employ a certain level of simplification, such that friction will be considered only for some bodies. This friction is reflected in the dissipative energy formulation.

The total kinetic energy of the mechanism $E_{K_{t o t}}$ equals the sum of kinetic energies of individual bodies $\sum_{i=1}^{n} E_{K_{i}}$ as follows:

$$
E_{K_{t o t}}=\sum_{i=1}^{n} E_{K_{i}}, i=1,2, \ldots, n,
$$

where $n$ is the number of bodies.

Hence, the resulting kinetic energy of the mechanism is given by the following formula:

$$
E_{K_{t o t}}=\frac{1}{2} \cdot I_{1} \cdot \dot{\varphi}_{1}^{2}+\frac{1}{2} \cdot m_{1} \cdot \dot{x}_{1}^{2}+\frac{1}{2} \cdot I_{2} \cdot \dot{\varphi}_{1}^{2}+\frac{1}{2} \cdot I_{3} \cdot \dot{\varphi}_{1}^{2}+\frac{1}{2} \cdot I_{4} \cdot \dot{\varphi}_{2}^{2}+\frac{1}{2} \cdot I_{5} \cdot \dot{\varphi}_{3}^{2}+\frac{1}{2} \cdot I_{6} \cdot \dot{\varphi}_{4}^{2},
$$

where $I_{1}, I_{2}, I_{3}, I_{4}, I_{5}$, and $I_{6}$ are the moments of inertia of individual bodies performing rotational movements (Figure $7 \mathrm{a}$ ); $m_{1}$ is the mass of the body performing a translation 
movement; deviations $\varphi_{1}, \varphi_{2}, \varphi_{3}$, and $\varphi_{4}$ are the angular coordinates of individual bodies (Figure 7a); and $x_{1}$ is a translation coordinate (Figure 7a).

With the input (generalized coordinate $\varphi_{2}$ ) and the output (generalized coordinate $\left.\varphi_{1}\right)$, a gearbox with a ratio of $i=2$ can be mounted. The gear ratio is expressed as follows:

$$
i=\frac{\varphi_{2}}{\varphi_{1}} .
$$

Then, we can express the generalized coordinate $\varphi_{2}$ using the generalized coordinate $\varphi_{1}$. Hence, the kinetic energy (Equation (3)) takes the following form:

$$
E_{K_{t o t}}=\frac{1}{2} \cdot I_{1} \cdot \dot{\varphi}_{1}^{2}+\frac{1}{2} \cdot m_{1} \cdot \dot{x}_{1}^{2}+\frac{1}{2} \cdot I_{2} \cdot \dot{\varphi}_{1}^{2}+\frac{1}{2} \cdot I_{3} \cdot \dot{\varphi}_{1}^{2}+\frac{1}{2} \cdot I_{4} \cdot\left(2 \cdot \dot{\varphi}_{1}\right)^{2}+\frac{1}{2} \cdot I_{5} \cdot \dot{\varphi}_{3}^{2}+\frac{1}{2} \cdot I_{6} \cdot \dot{\varphi}_{4}^{2} \text {. }
$$

The final form of the total kinetic energy $E_{K_{\text {tot }}}$ is as follows:

$$
E_{K_{\text {tot }}}=\frac{1}{2} \cdot\left[m_{1} \cdot \dot{x}_{1}^{2}+\left(I_{1}+I_{2}+I_{3}+4 \cdot I_{4}\right) \cdot \dot{\varphi}_{1}^{2}+I_{5} \cdot \dot{\varphi}_{3}^{2}+\frac{1}{2} \cdot I_{6} \cdot \dot{\varphi}_{4}^{2}\right],
$$

where the relation in round brackets represents the reduced moment of inertia $I_{1 \text { red }}$ for the mutual shaft-in this case, shaft 1 . This moment can be written in the following form:

$$
I_{1 \text { red }}=\left(I_{1}+I_{2}+I_{3}+4 \cdot I_{4}\right)
$$

Furthermore, the dissipative energy represents the sum of the energies, which include energies wasted by the system itself. In our case, we consider the viscous friction in the system, namely, the friction of the bodies moving rectilinearly (with the generalized coordinate $x_{1}$ ) as well as the bodies performing rotating movements described by the generalized coordinate $\varphi_{1}$. Friction-related phenomena are included in coefficients $b_{1}, b_{2}$, $b_{3}$, and $b_{4}$. Hence, the dissipative energy assumes the following form:

$$
E_{D}=\frac{1}{2} \cdot\left[b_{1} \cdot \dot{x}_{1}^{2}+b_{2} \cdot \dot{\varphi}_{2}^{2}+b_{3} \cdot \dot{\varphi}_{3}^{2}+b_{4} \cdot \dot{\varphi}_{4}^{2}\right]=\frac{1}{2} \cdot\left[b_{1} \cdot \dot{x}_{1}^{2}+4 \cdot b_{2} \cdot \dot{\varphi}_{1}^{2}+b_{3} \cdot \dot{\varphi}_{3}^{2}+b_{4} \cdot \dot{\varphi}_{4}^{2}\right] .
$$

Considering a torsion stiffness of the individual sections of shafts and a connecting rod, we can derive the potential energy of the system. For this purpose, we use the following equation:

$$
E_{P}=\frac{1}{2} \cdot\left[k_{1} \cdot x_{1}^{2}+k_{2} \cdot\left(\varphi_{3}-\varphi_{2}\right)^{2}+k_{3} \cdot\left(\varphi_{1}-\varphi_{4}\right)^{2}\right]=\frac{1}{2} \cdot\left[k_{1} \cdot x_{1}^{2}+k_{2} \cdot\left(\varphi_{3}-2 \cdot \varphi_{1}\right)^{2}+k_{3} \cdot\left(\varphi_{1}-\varphi_{4}\right)^{2}\right]
$$

where $k_{1}$ is the stiffness of the connecting rod, and $k_{2}$ and $k_{3}$ are the torsion stiffness values of individual parts of the shafts (Figure 7a).

Next, we can proceed to derive the individual energies written in Equations (6), (8) and (9). Derivations are performed based on the general Equation (1), whereas the derivations themselves are not included.

The final system of equations of motion for the solved mechanical system can then be written as follows:

$$
\begin{array}{r}
m_{1} \cdot \ddot{x}_{1}+b_{1} \cdot \dot{x}_{1}+k_{1} \cdot x_{1}=F(t) \\
I_{s} \cdot \ddot{\varphi}_{1}+2 \cdot b_{2} \cdot \dot{\varphi}_{1}+\left(4 \cdot k_{2}+k_{3}\right) \cdot \varphi_{1}-2 \cdot k_{3} \cdot \varphi_{3}-k_{3} \cdot \varphi_{4}=0 \\
I_{5} \cdot \ddot{\varphi}_{3}+b_{3} \cdot \dot{\varphi}_{3}-2 \cdot k_{2} \cdot \varphi_{1}+k_{2} \cdot \varphi_{3}=0 \\
I_{6} \cdot \ddot{\varphi}_{4}+b_{4} \cdot \dot{\varphi}_{4}-k_{3} \cdot \varphi_{1}+k_{3} \cdot \varphi_{4}=0
\end{array}
$$

Additionally, the related matrix form is 


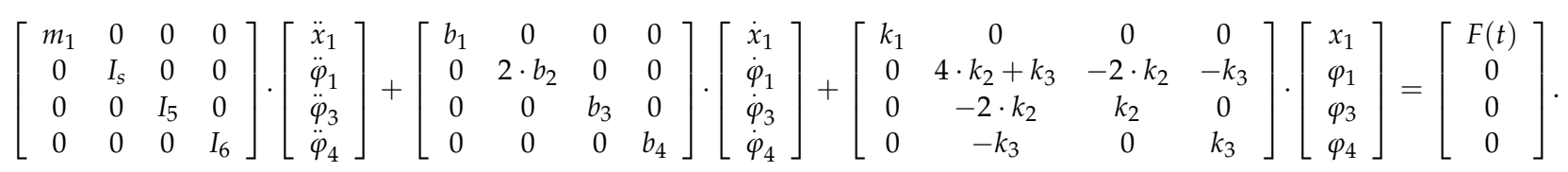

Adopting the known terminology,

$$
\boldsymbol{M}=\left[\begin{array}{cccc}
m_{1} & 0 & 0 & 0 \\
0 & I_{s} & 0 & 0 \\
0 & 0 & I_{5} & 0 \\
0 & 0 & 0 & I_{6}
\end{array}\right]
$$

is the mass matrix,

$$
\boldsymbol{B}=\left[\begin{array}{cccc}
b_{1} & 0 & 0 & 0 \\
0 & 2 \cdot b_{2} & 0 & 0 \\
0 & 0 & b_{3} & 0 \\
0 & 0 & 0 & b_{4}
\end{array}\right]
$$

is the damping matrix,

$$
\boldsymbol{K}=\left[\begin{array}{cccc}
k_{1} & 0 & 0 & 0 \\
0 & 4 \cdot k_{2}+k_{3} & -2 \cdot k_{2} & -k_{3} \\
0 & -2 \cdot k_{2} & k_{2} & 0 \\
0 & -k_{3} & 0 & k_{3}
\end{array}\right]
$$

is the stiffness matrix, and

$$
\begin{aligned}
\ddot{x} & =\left[\begin{array}{llll}
\ddot{x}_{1} & \ddot{\varphi}_{1} & \ddot{\varphi}_{3} & \ddot{\varphi}_{4}
\end{array}\right]^{T}, \\
\dot{x} & =\left[\begin{array}{llll}
\dot{x}_{1} & \dot{\varphi}_{1} & \dot{\varphi}_{3} & \dot{\varphi}_{4}
\end{array}\right]^{T}, \\
\boldsymbol{x} & =\left[\begin{array}{llll}
x_{1} & \varphi_{1} & \varphi_{3} & \varphi_{4}
\end{array}\right]^{T}
\end{aligned}
$$

are vectors of generalized accelerations and velocities, respectively. Furthermore,

$$
\boldsymbol{F}=\left[\begin{array}{cccc}
F(t) & 0 & 0 & 0
\end{array}\right]^{T}
$$

is the vector of external loads or the excitation of the system. As this engine is assumed to be powered by compressed air, the force $F(t)$ in vector $F$ represents the vector's time function. However, we do not yet know how to mathematically describe this force.

If we take into account the dimensions and functions of the engine mechanism, we can consider certain simplifications. First, the plate, switchboard, and cylinders are considered as one functional unit with a mutual moment of inertia $I_{S}$ (i.e., the reduced moment of inertia in Equation (7)). Due to the very short lengths of the shafts, the torsion stiffness is very high. Consequently, the relative rotation of the shaft ends, i.e., torsion deformation, can be neglected. We also consider the very high stiffness of the connecting rod. As a result of this high stiffness, the differences in the deformations of individual bodies described by Equation (9) are zero; therefore, the potential energy of this system is also considered zero. These simplifications do not have significant effects on the output variables (Figure $7 \mathrm{~b}$ ).

Hence, the initial generalized coordinates $\varphi_{1}, \varphi_{2}, \varphi_{3}$, and $\varphi_{4}$ are identical to the generalized coordinate $\varphi_{1}$ (i.e., $\varphi_{1}=\varphi_{2}=\varphi_{3}=\varphi_{4}$ ). Therefore, these simplifications lead to modified kinetic energy $E_{K_{\bmod }}$ as follows:

$$
E_{K_{\mathrm{mod}}}=\frac{1}{2} \cdot m_{1} \cdot \dot{x}_{1}^{2}+\frac{1}{2} \cdot I_{p} \cdot \dot{\varphi}_{1}^{2}+\frac{1}{2} \cdot I_{s} \cdot \dot{\varphi}_{1}^{2}+\frac{1}{2} \cdot I_{i} \cdot\left(2 \cdot \dot{\varphi}_{1}\right)^{2}+\frac{1}{2} \cdot I_{v 1} \cdot \dot{\varphi}_{1}^{2}+\frac{1}{2} \cdot I_{v 2} \cdot\left(2 \cdot \dot{\varphi}_{1}\right)^{2} \text {. }
$$


We can then modify Equation (19) as follows:

$$
E_{K_{\text {mod }}}=\frac{1}{2} \cdot\left[m_{1} \cdot \dot{x}_{1}^{2}+\left(I_{p}+I_{s}+4 \cdot I_{i}+I_{v 1}+4 \cdot I_{v 2}\right) \cdot \dot{\varphi}_{1}^{2}\right] .
$$

The expression in round brackets is called the reduced moment of inertia, which we mark as $I_{\text {red }}$. The final form of the modified kinetic energy $E_{K_{\text {mod }}}$ is then written as

$$
E_{K_{\mathrm{mod}}}=\frac{1}{2} \cdot\left[m_{1} \cdot \dot{x}_{1}^{2}+I_{\text {red }} \cdot \dot{\varphi}_{1}^{2}\right] .
$$

As mentioned above, the potential energy equals zero. Considering only the modified kinetic energy and the dissipative energy and performing derivations according to Equation (1), we can obtain simplified equations of motion:

$$
\begin{array}{r}
m_{1} \cdot \ddot{x}_{1}+b_{1} \cdot \dot{x}_{1}=F(t) \\
I_{\text {red }} \cdot \ddot{\varphi}_{1}+\left(b_{1}+2 \cdot b_{2}\right) \cdot \dot{\varphi}_{1}=0
\end{array} .
$$

The matrix form of the equations of motion written in Equation (22) is

$$
\left[\begin{array}{cc}
m_{1} & 0 \\
0 & I_{\text {red }}
\end{array}\right] \cdot\left[\begin{array}{c}
\ddot{x}_{1} \\
\ddot{\varphi}_{1}
\end{array}\right]+\left[\begin{array}{cc}
b_{1} & 0 \\
0 & b_{1}+2 \cdot b_{2}
\end{array}\right] \cdot\left[\begin{array}{c}
\dot{x}_{1} \\
\dot{\varphi}_{1}
\end{array}\right]=\left[\begin{array}{c}
F(t) \\
0
\end{array}\right]
$$

where

$$
\boldsymbol{M}_{\mathrm{mod}}=\left[\begin{array}{cc}
m_{1} & 0 \\
0 & I_{\text {red }}
\end{array}\right]
$$

is the modified mass matrix;

$$
\boldsymbol{B}_{\text {mod }}=\left[\begin{array}{cc}
b_{1} & 0 \\
0 & b_{1}+2 \cdot b_{2}
\end{array}\right]
$$

is the modified damping matrix;

$$
\begin{aligned}
& \ddot{x}_{\text {mod }}=\left[\begin{array}{ll}
\ddot{x}_{1} & \ddot{\varphi}_{1}
\end{array}\right]^{T} \\
& \dot{x}_{\text {mod }}=\left[\begin{array}{ll}
\dot{x}_{1} & \dot{\varphi}_{1}
\end{array}\right]^{T}
\end{aligned}
$$

are the modified vectors of accelerations and velocities, respectively; and

$$
F_{\text {mod }}=\left[\begin{array}{ll}
F(t) & 0
\end{array}\right]
$$

is the modified vector of excitation for the engine mechanism.

Preliminary masses and moments of inertia were obtained via CAD (Computer-Aided Design) models created in the Autodesk Inventor software. The exact values of these parameters are measured by weighing when the prototype is produced. All strength values of the related components will be determined in the near future. However, the kinematic analyses and simulations of the engine can already be performed based on the data obtained. Therefore, the unit mass and inertia quantities were defined in the present study. The results of the simulation computations are presented in the next section.

\section{Results}

A multibody model (MBS model) of the engine with rotating cylinders was created in the Simpack software package (Dassault Systèmes, Vélizy-Villacoublay, France; Figure 8a). Figure $8 \mathrm{~b}$ shows the detail of joints defined in the Simpack software package. The model includes both mechanical and kinematic joints. 


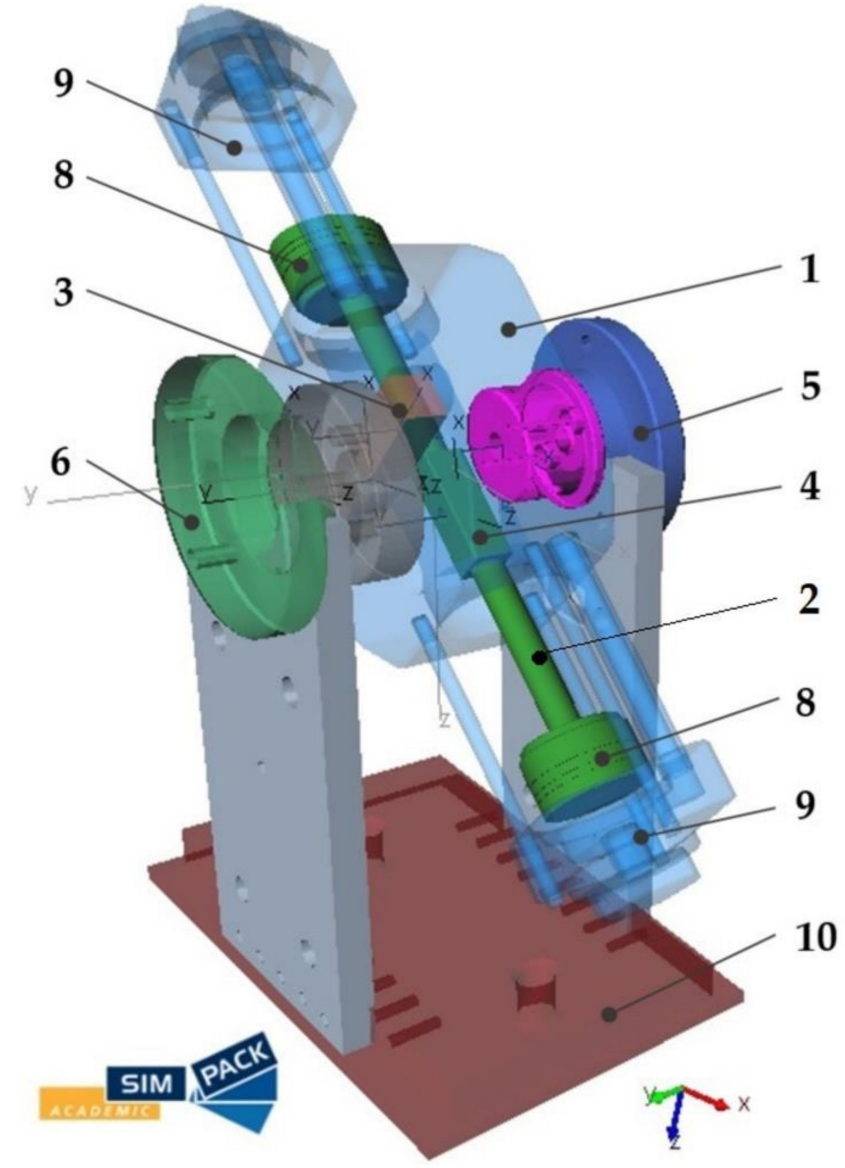

(a)

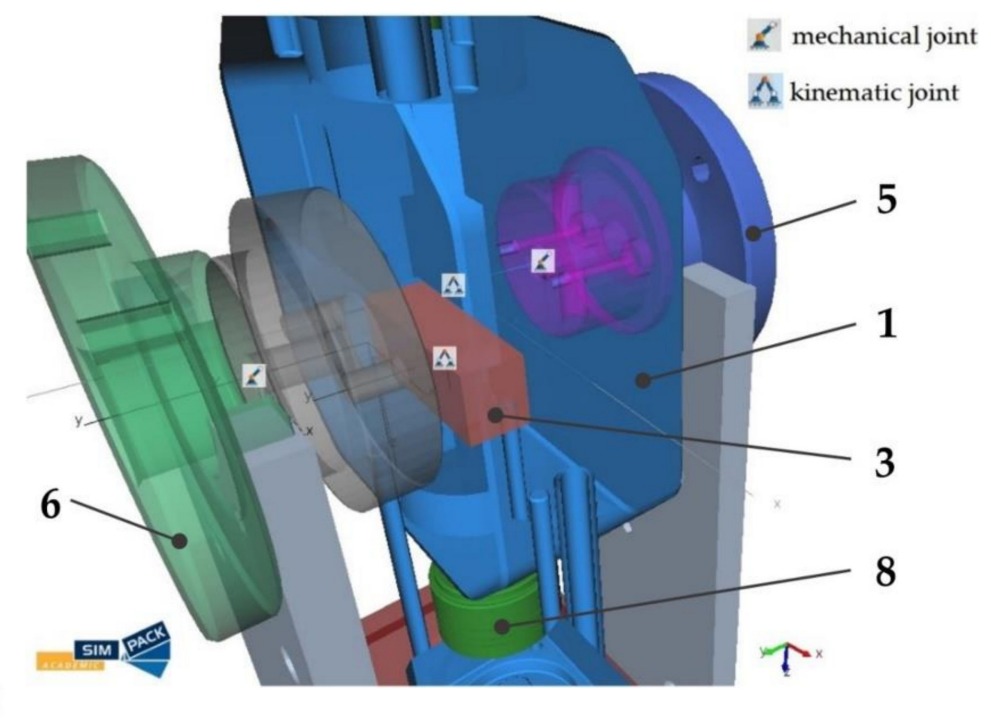

(b)

Figure 8. A multibody model of the engine in the Simpack software package (a), the detail of joints (mechanical and kinematic joints) (b): 1-a plate, 2-a connecting rod, 3-an auxiliary stone, 4-a stone, 5-a first output, 6-a second output, 8-pistons, 9-heads of cylinders, 10-a stand.

Simpack is a widely used MBS software package that can render models of various mechanical systems, including simplified subsystems of vehicles, such as engines [39] and gearboxes [40,41], parts of chassis and suspension systems, entire vehicles [42,43], and sets of vehicles or other mechanisms $[44,45]$. Simpack works using numerical methods in which a mathematical model describing the dynamic behavior of a mechanical system can be derived automatically.

Firstly, markers for placing individual components and defining mechanical and kinematical couplings were defined. Individual bodies of the solved mechanism were then connected to each other by the corresponding joints and constraints. In the created MBS model, all bodies were considered rigid. This means that the dynamic properties of the bodies were fully described by their masses, moments of inertia, and centers of gravity. For better visualization, the geometries of the bodies were defined by models imported from the Autodesk Inventor software (Figure 4). However, as the definitions of materials of individual bodies require one to perform strength analyses, the motion properties of the engine are analyzed in terms of the engine's kinematics, and the mass and inertia properties are defined as units.

Based on the created computational models and their parameters, we selected waveforms for some kinematic quantities of the engine. The engine waveforms were compared, achieving results for the two approaches:

- The mathematical model derived in Section 3 and solved in MATLAB (MathWorks, Natick, MA, USA); 
- The numerical model is represented by the MBS model created in the Simpack software (Figure 8).

The MBS model consists only of rigid bodies in which all the linking elements, such as shafts, are also considered rigid. Therefore, the compared numerical model developed in MATLAB was based on equations of motion in compliance with Equation (23). It should be noted that calculations were carried out for unit masses and inertia properties because current calculations are focused on analyses of the kinematic properties of the engine mechanism.

The resulting waveforms and graphs were processed in MATLAB to compare them within individual figures. From all the results, we selected waveforms for some kinematic quantities, including for a functional block (Figure 9), a piston mechanism (Figures 10 and 11), the first output, the second output, and a switchboard (Figures 12 and 13).

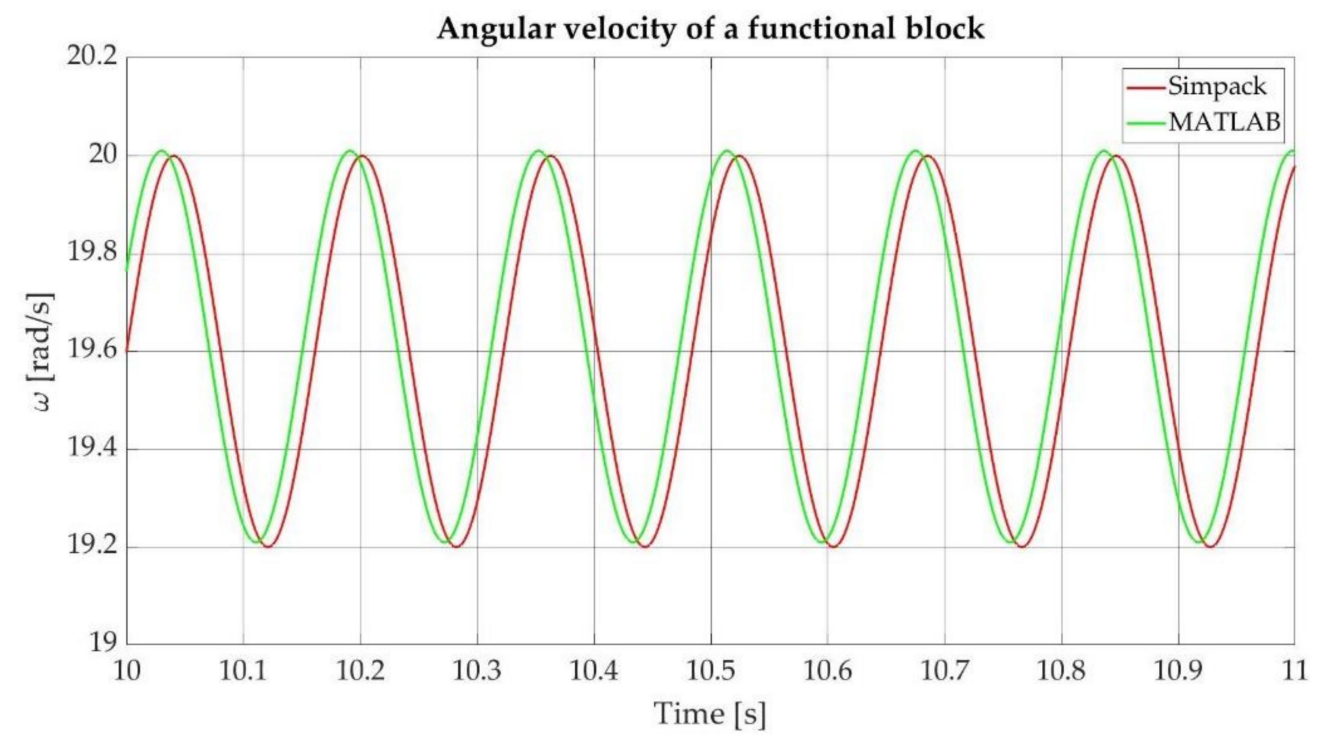

Figure 9. Angular velocity of a functional block-a comparison between the results from Simpack and MATLAB.

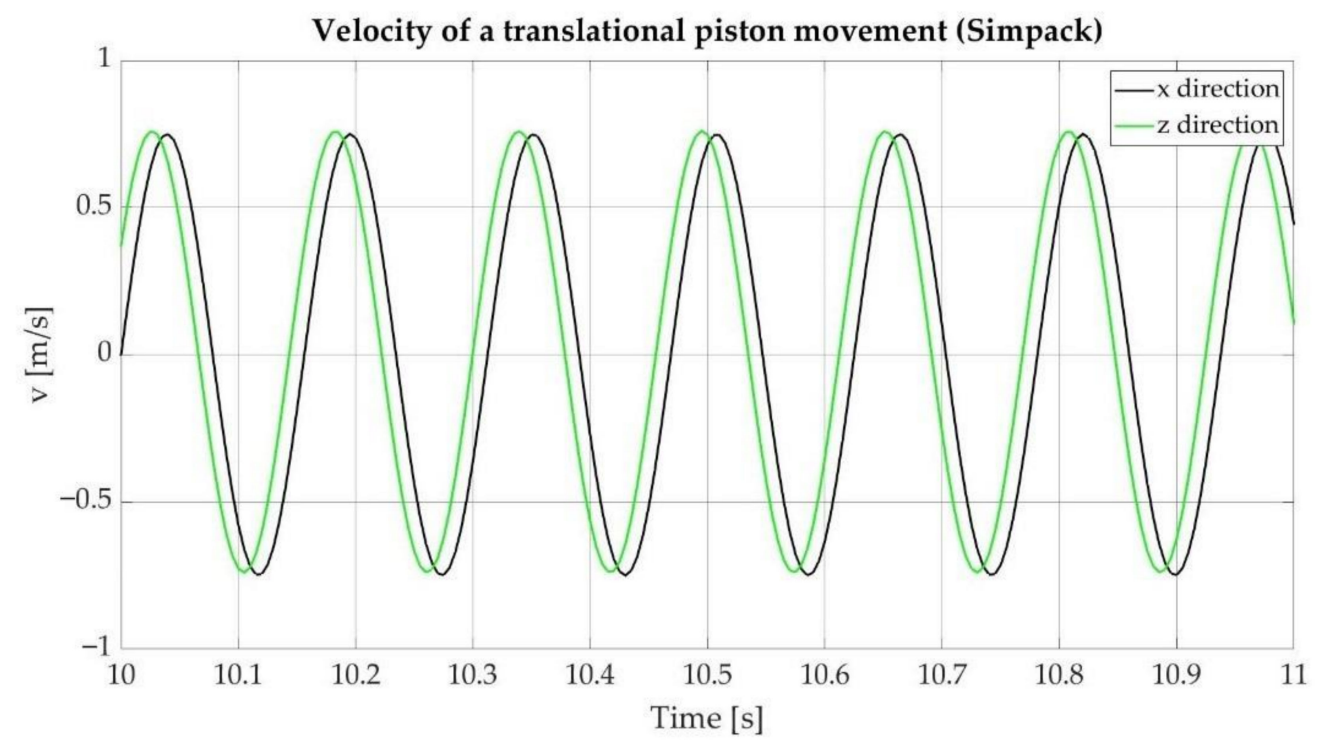

Figure 10. Translational velocity of pistons—results from Simpack. 


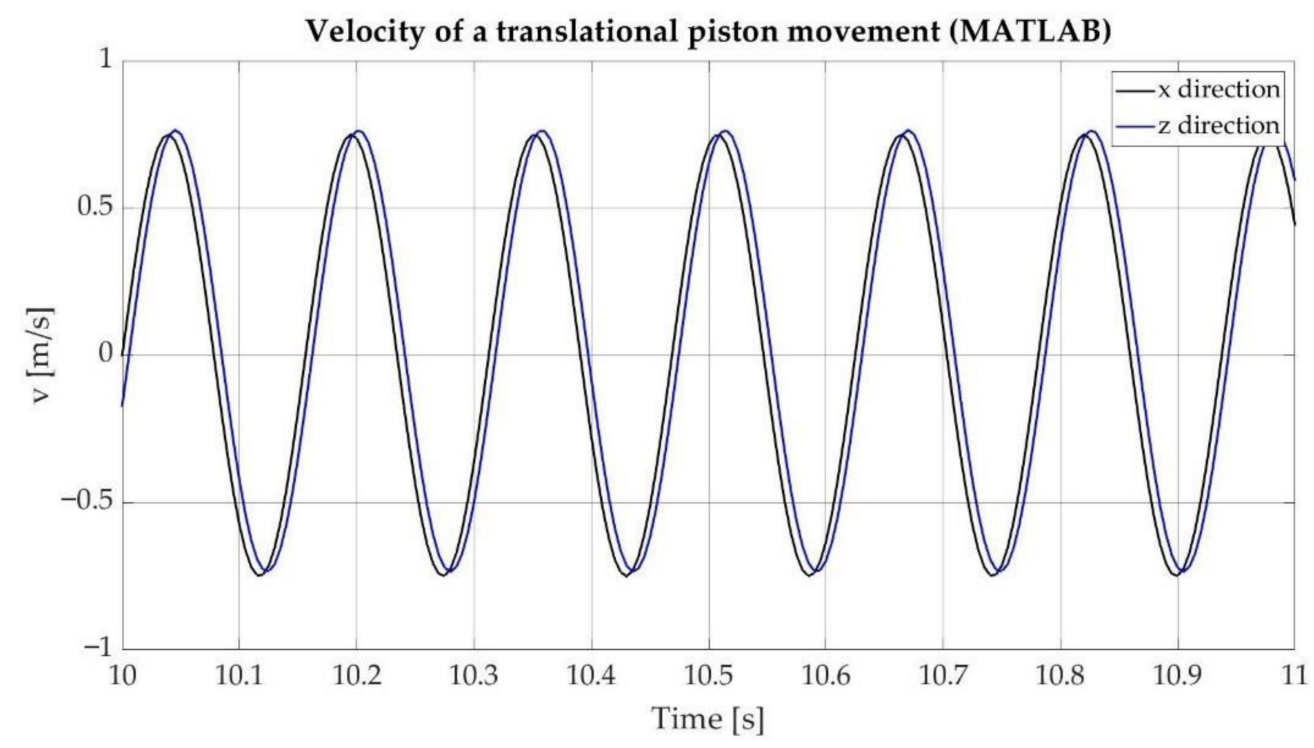

Figure 11. Translational velocity of pistons-results from MATLAB.

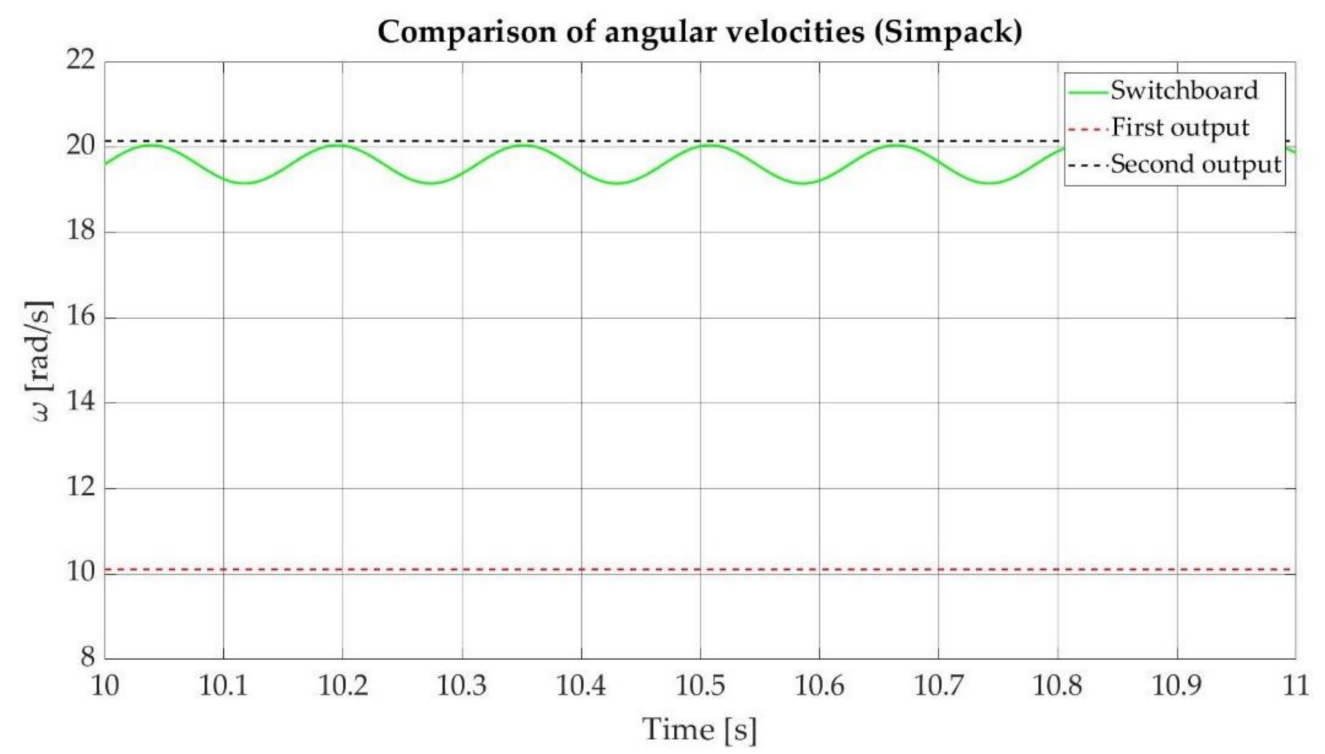

Figure 12. Comparison of the angular velocities of the first output, second output, and switchboardresults from Simpack.

Here, the angular velocity of the functional block is $\varphi_{1}=20 \mathrm{rad} \cdot \mathrm{s}^{-1}$, the angular velocity of the first output is $\varphi_{2}=10 \mathrm{rad} \cdot \mathrm{s}^{-1}$, the angular velocity of the second output due to the technical solution is $\varphi_{1}=20 \mathrm{rad} \cdot \mathrm{s}^{-1}$, and the gear ratio is $i_{g}=0.5$.

Figure 9 shows the waveforms for the angular velocity of the functional block of the engine mechanism obtained from Simpack and MATLAB. Both results correspond to each other with only small deviations. Figure 9 shows the waveform of the angular velocity of the functional block. Here, the velocity oscillates despite the chosen constant value. This oscillation is due to the compound motion of the pistons, which perform both rotational and translational movement at the same time. This compound movement changes the position of the center of gravity in the mechanical system. 


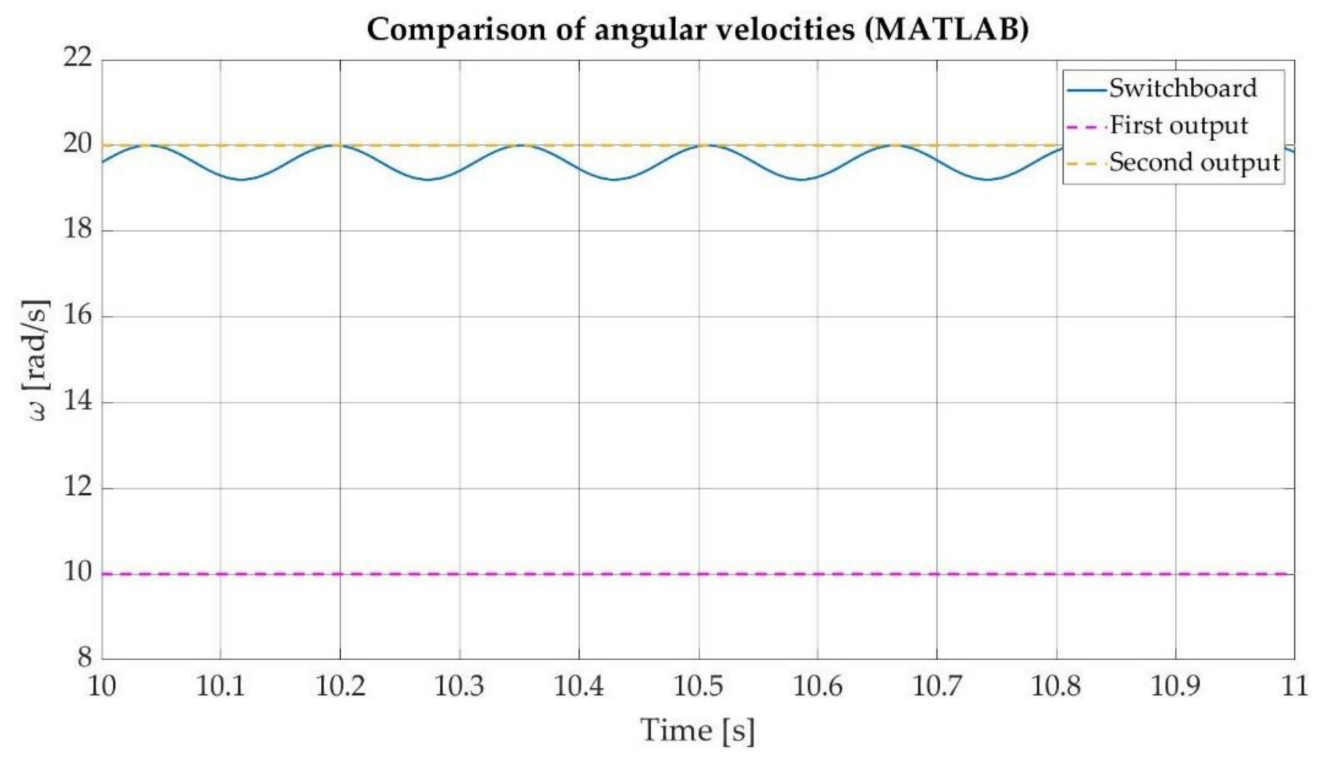

Figure 13. Comparison of the angular velocities of the first output, second output, and switchboardresults from MATLAB.

The velocity of the pistons obtained from the Simpack software is illustrated in Figure 10, and the velocity obtained from MATLAB is shown in Figure 11. The results of velocity are shown for both the $x$ and $z$ axes. As can be seen, this motion is harmonic in both directions. Comparing the results, the amplitudes and frequencies of the investigated movements are similar in both the Simpack (Figure 10) and MATLAB (Figure 11) outputs.

Lastly, Figures 12 and 13 show the time dependence of the angular velocities of the first and second outputs and the switchboard under constant rotations. These values are constant because, for the outputs, the position of the center of gravity is constant. Further, the angular velocity of the switchboard oscillates according to the harmonic function. When compared in more detail, the amplitudes and frequency are very similar to the waveforms of the angular velocity of the functional block. The angular velocities of the first and second outputs are constant, and these values are the same as those of the angular velocity of the functional block.

\section{Discussion}

The results of this study show that the mathematical model of the engine mechanism movement described by the derived equations of motion can be verified by the numerical model created in the MBS software. The waveforms of the observed kinematic quantities indicate only slight deviations from each other. Figure 9 contains the comparison of the angular velocities of the functional block for the MATLAB and Simpack software. At the mean value of $19.60 \mathrm{rad} / \mathrm{s}$, the amplitude obtained by the MATLAB was $20.01 \mathrm{rad} / \mathrm{s}$ and by the Simpack was $20.00 \mathrm{rad} / \mathrm{s}$. Further, the translational velocity of the pistons in $x$ and $z$ directions calculated by both software were analyzed (Figures 10 and 11). Firstly, we evaluated the results of the translational velocity for the $x$ direction. Its amplitude calculated by the MATLAB resulted in the value of $0.7650 \mathrm{~m} / \mathrm{s}$. The Simpack gave an amplitude of $0.7599 \mathrm{~m} / \mathrm{s}$. Their difference represents the value of $0.0051 \mathrm{~m} / \mathrm{s}$. On the other hand, the amplitude of the translational velocity of the pistons in the $z$ direction is exactly the same, and that of $0.7499 \mathrm{~m} / \mathrm{s}$. Thus, the difference in the translational velocity of the piston movement in the $z$ direction equals zero. Finally, Figures 12 and 13 include waveforms of the angular velocities of the first output, second output, and switchboard. Again, there are compared results obtained by the MATLAB and Simpack software. As it can be seen, the angular velocity of the first and the second outputs are constant during the entire time interval. The value of the angular velocity of the first output calculated by the MATLAB is $10.00 \mathrm{rad} / \mathrm{s}$ and calculated by the Simpack is $10.11 \mathrm{rad} / \mathrm{s}$. The angular 
velocity of the second output is $20.00 \mathrm{rad} / \mathrm{s}$ for the MATLAB and of $20.15 \mathrm{rad} / \mathrm{s}$ for the Simpack. From this, the difference of angular velocities of the first output is $0.11 \mathrm{rad} / \mathrm{s}$ and for the second output is $0.15 \mathrm{rad} / \mathrm{s}$. As the last compared kinematic quantity, the angular velocity of the switchboard was chosen. In contrast to other angular velocities shown in Figures 12 and 13, the angular velocity of the switchboard has the sinusoidal waveforms for both the MATLAB and Simpack software. The mean value is the same for both software, namely $19.60 \mathrm{rad} / \mathrm{s}$. However, the amplitude obtained by the MATLAB is $0.40 \mathrm{rad} / \mathrm{s}$ and by Simpack is $0.45 \mathrm{rad} / \mathrm{s}$. From this, the difference of the amplitudes is $0.05 \mathrm{rad} / \mathrm{s}$. Based on these results, we conclude that both models were set up properly and could be used for further research.

In addition, the results of complex simulations performed by the multibody program Simpack did not show the presence of a singularity in the constructed mechanism. From the compiled analytical and numerical model, it is, therefore, possible to proceed to the construction of a real prototype (Figure 14) without the danger of a malfunction resulting from the selected geometry. Up to this time, the prototype is ready and fully functional. Future research will focus on experimental measurement and tuning of the influence of kinematic parameters on engine dynamics. The prototype will be analyzed by numerical simulations to verify strength parameters, maximum speed, operating pressure, etc. A proposal of the optimal material selection of individual engine components will be presented.

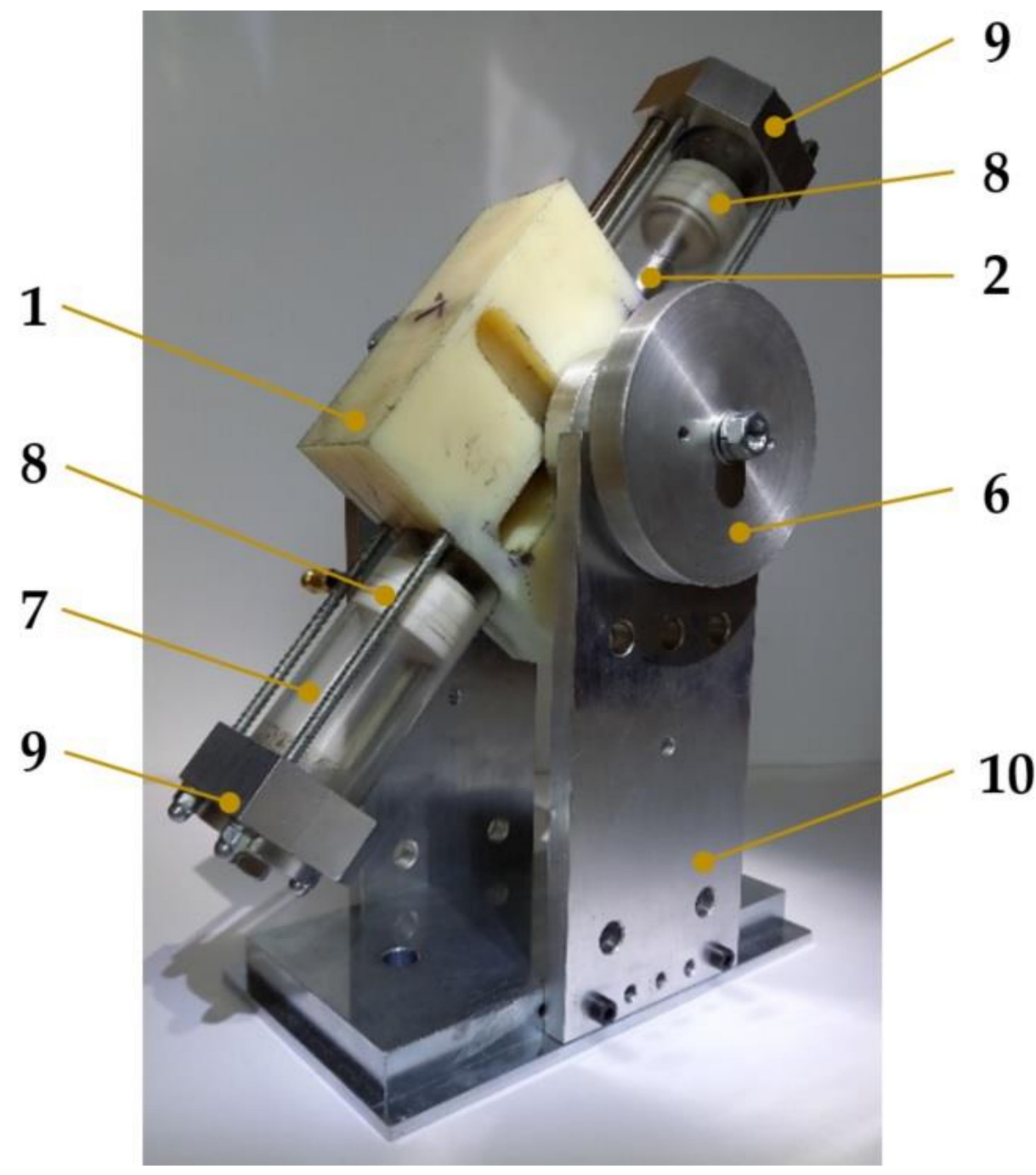

Figure 14. A real prototype of the compressed air engine design: 1-a plate, 2-a connecting rod, 6-a second output, 7-cylinders, 8-pistons, 9-heads of cylinders, 10-a stand. 
The waveforms of the individual kinematic quantities show output characteristics similar to those of the waveforms of the output quantities on the crankshaft of a conventional combustion engine. Therefore, a flywheel should be applied to smooth the run of the resulting torque. This flywheel will ensure that the torque is regular and that the transmission of this torque will not cause excessive vibrations of other related elements [1,2,9,12].

Finally, here are assessed the potential frictional losses of this non-conventional engine mechanism design. The designed model is symmetrical with six kinematic couples as following: two pistons in cylinders-two translational couplings; stones in a plate-two general couplings; bearings lengths-two rolling couplings. For this time, the manufactured prototype is determined for pedagogical purposes; therefore, cylinders are made of transparent plastic material PMMA (polymethyl methacrylate), and pistons are made of PTFE (polytetrafluoroethylene). The friction coefficient is of $f_{A p}=0.40$ [46]. Further, copper stones are guided in a technical plastic PA6G (cast nylon), and their friction coefficient is of $f_{B p}=0.80$ [46]. Rolling coefficients of two rolling bearings are assumed of $f_{C}=0.02$ [46]. Efficiency coefficients are inverse values. As a result, the total efficiency of the designed engine with transparent plastic cylinders is very small.

Therefore, it is necessary to take into account the use of other more suitable materials for the real application of the designed engine. The plastic components would be surely made of metal material. We assume that steel would be a suitable material. Then, we can assume different and mainly lower values of friction coefficients of kinematic couplings. If the cylinders were made of steel, the friction coefficient would have a value of $f_{A s}=0.04$, and the friction coefficient between a metal plate and stones would be assumed of $f_{B s}$ $=0.08$ [46]. Thus, the total mechanical efficiency of the engine mechanism with metal components $\eta_{T s}$ is calculated as follows:

$$
\eta_{T s}=\eta_{A s}^{\prime} \cdot \eta_{A s}^{\prime \prime} \cdot \eta_{B s}^{\prime} \cdot \eta_{B s}^{\prime \prime} \cdot \eta_{C}^{\prime} \cdot \eta_{C}^{\prime \prime}
$$

where $\eta_{A s}^{\prime} \eta_{B s}^{\prime}$ and $\eta_{C}^{\prime}$ are efficiency coefficients of friction couples described above on one side of the engine and $\eta^{\prime \prime} A_{s}, \eta^{\prime \prime}{ }_{B s}$ and $\eta_{C}^{\prime}$ are efficiency coefficients of friction couples described above on the other side. The upper indices designate steel. Their values are assumed as follows: $\eta_{A s}^{\prime}=\eta^{\prime \prime}{ }_{A s}=0.96, \eta_{B s}^{\prime}=\eta_{B s}^{\prime \prime}=0.92$ and $\eta_{C}^{\prime}=\eta^{\prime \prime}{ }_{C}=0.98$. Substituting these values, we obtain the total efficiency of the mechanism with plastic cylinders:

$$
\eta_{T s}=0.96 \cdot 0.96 \cdot 0.92 \cdot 0.92 \cdot 0.98 \cdot 0.98=0.749153 \doteq 0.75 \text {. }
$$

In a percentage, the total efficiency of the engine mechanism with the metal components is $75 \%$.

When we compare the preliminarily calculated value with the mechanical efficiency of conventional combustion engines, it is comparable with ignition engines, which efficiency is usually in the range of $75 \%$ to $92 \%[47,48]$, but it is better compared with the mechanical efficiency of diesel engines, which efficiency is in the range of $70 \%$ to $87 \%[47,48]$. Our calculated value is still assumed. More accurate results will be revealed after more detailed analyses of the mechanical properties of individual components and after proper choice of the used materials for the final production of the engine.

In terms of thermodynamics, the designed engine uses rotating cylinders. It means that the engine could be cooled by air similarly to air-cooled engines. However, we assume that this non-conventional engine could not use a fan. The real efficiency of cooling could be assessed after experimental tests.

Future research activities will focus on developing more advanced models of the engine mechanism. For these models, we must determine all the geometrical parameters of all structural units of the engine to dynamically analyze a real engine and obtain relevant results. However, this condition is necessarily preceded by calculating the strength and verifying the applicability of the most commonly loaded components. Subsequently, it will be possible to create state equations that can describe the effects of the input medium (gas-i.e., compressed air) on the input rotations of the engine, including all passive forces 
and resistance moments. The friction coefficients of the kinematic couples will then be determined based on the table values. Future research will also focus on creating a real prototype of the engine. With this prototype, it will be possible to optimize the final design of the engine, which could be used for commercial applications. The desirable dynamic MBS model would also contain flexible bodies to represent the real behavior of the prototype better.

It can be said that the biggest anticipated advantage of a designed engine in comparison with other conventional and unconventional engines will be its production cost and higher overall efficiency. The real prototype in Figure 14 had an entry cost of less than EUR 200. In the future, it will be valuable to verify a specific application of another design and compare it with the given solution while maintaining the security that will result from the prepared static and dynamic calculations of real components. Therefore, these will also need to be designed.

\section{Conclusions}

In this study:

- We designed and patented a technical solution for a new non-conventional engine; this solution could be applied to a wide range of mechanisms;

- We derived and created a mathematical model of the engine mechanism using Lagrange's equations of motion of the second kind, as well as a numerical model in the Simpack commercial software. These mathematical models represent a very important development in the creation of more detailed models to describe the dependence of the driving medium pressure and the values of the maximal rotations. All presented data are necessary to regulate the engine for loading and use;

- We performed MBS simulations. The results of the chosen kinematic quantities for these numerical simulations demonstrated the relevant effects of the gear mechanism;

- Our results showed that the mathematical model of the engine mechanism described by the equations of motion and the multibody model created in the MBS software were very similar to each other. This indicates the correctness of the chosen procedure and the two models;

- A real prototype was also made in the laboratory conditions, and its functionality was verified, which can be considered as the greatest contribution of the possibility of commercial use;

- $\quad$ The created mathematical model and subsequent computational models and analyses have proven that the mathematical description of the engine mechanism is carried out correctly. As the engine design is still being developed, these materials will serve as the key background for further research. It will allow to predict dynamic properties of the engine under various operational conditions, to detect possible deficiencies at the extreme operational conditions, e.g., to high input pressure, high engine speed, to avoid excessive vibration, which could be caused by imbalances and other technical issues. Moreover, the virtual models allow to optimize the parameters of the engine without having a real product, i.e., this really saves production costs, shortens time of development as well as makes it easier to perform any modifications during the development process of the final engine prototype.

In future research, we intend to build a real prototype of the engine and then effectively optimize the final design of this engine to compete against conventional engines.

Author Contributions: Conceptualization, J.D. and M.B.; methodology, J.D., M.B. and M.S.; software, J.D. and P.Š.; validation, J.D. and M.B.; formal analysis, M.S. and P.Š.; investigation, J.D., M.B. and M.S; resources, J.D. and P.Š.; writing — original draft preparation, J.D. and M.B.; writing-review and editing, J.D. and M.S.; visualization, J.D. and M.B.; supervision, M.S. All authors have read and agreed to the published version of the manuscript.

Funding: This research was supported by the Cultural and Educational Grant Agency of the Ministry of Education of the Slovak Republic in project No. KEGA 023ŽU-4/2020: Development of advanced 
virtual models for studying and investigation of transport means operation characteristics. This research was supported by the project VEGA 1/0073/19: Computer modeling and development of algorithms for evaluating the reliability of composite structural elements produced by the additive technology based on Onyx for the purpose of optimal design.

Institutional Review Board Statement: Not applicable.

Informed Consent Statement: Not applicable.

Data Availability Statement: Not applicable.

Conflicts of Interest: The authors declare no conflict of interest.

\section{Nomenclature}

\begin{tabular}{|c|c|}
\hline CAD & Computer-aided design \\
\hline PA6G & Cast nylon \\
\hline PMMA & Polymethyl methacrylate \\
\hline PTFE & Polytetrafluoroethylene \\
\hline$B$ & Damping matrix \\
\hline$B_{\text {mod }}$ & Modified damping matrix \\
\hline$b_{1}$ & Damping coefficient of a first output \\
\hline$b_{2}$ & Damping coefficient of a second output \\
\hline$E_{D}$ & Dissipative energy \\
\hline$E_{K}$ & Kinetic energy \\
\hline$E_{K_{\text {mod }}}$ & Modified kinetic energy \\
\hline$E_{K_{\text {tot }}}$ & Total kinetic energy \\
\hline$E_{P}$ & Potential energy \\
\hline$F$ & External load \\
\hline$f_{A p}$ & Friction coefficient of plastic cylinders and pistons \\
\hline$f_{A s}$ & Friction coefficient of steel cylinders and pistons \\
\hline$f_{B p}$ & Friction coefficient of a plastic plate and stones \\
\hline$f_{B s}$ & Friction coefficient of a steel plate and pistons \\
\hline$f_{C}$ & Friction coefficient of bearings length \\
\hline$I_{1}, I_{2}, I_{3}, I_{4}, I_{5}, I_{6}$ & Moments of inertia of individual bodies \\
\hline$I_{1 \text { red }}$ & Reduced moment of inertia \\
\hline$I_{S}$ & Mutual moment of inertia \\
\hline$i$ & The number of degrees of freedom \\
\hline$i_{g}$ & The gear ratio \\
\hline K & Stiffness matrix \\
\hline$k_{1}, k_{2}, k_{3}, k_{4}$ & Stiffness of individual components \\
\hline$K_{\text {mod }}$ & Modified stiffness matrix \\
\hline$M$ & Mass matrix \\
\hline$M_{\text {mod }}$ & Modified mass matrix \\
\hline$m_{1}$ & Mass of the body 1 \\
\hline$n$ & Number of bodies \\
\hline$n_{1}$ & Rotations of a first output \\
\hline$n_{2}$ & Rotations of a second output \\
\hline$Q$ & Sum of the external loads \\
\hline$x, \dot{x}, \ddot{x}$ & Translational coordinate, velocity, acceleration \\
\hline$\varphi_{1}, \varphi_{2}$ & Angular deflections \\
\hline$\eta_{T s}$ & Total mechanical efficiency with steel components \\
\hline$\eta_{A s}^{\prime} \eta^{\prime \prime} A s$ & Mechanical efficiency of coupling steel cylinders-pistons, both sides \\
\hline$\eta_{B s}^{\prime}, \eta_{B S}^{\prime \prime}$ & Mechanical efficiency of coupling steel plate-stones, both sides \\
\hline$\eta_{C}^{\prime}, \eta_{C}^{\prime \prime}$ & Mechanical efficiency of bearings lengths-both sides \\
\hline
\end{tabular}

\section{References}

1. Van Basshuysen, R.; Schaefer, F. Internal Combustion Engine Handbook, 2nd ed.; SAE International: Warrendale, PA, USA, 2016.

2. Stone, R. Introduction to Internal Combustion Engines, 4th ed.; SAE International and Macmillan Press: London, UK, 2012.

3. Dobrodenka, P.; Lešinský, J. A Four-Stroke Engine with Opposite Pistons. Utility model SK3731 Y1, 21 January 2004. 
4. Baran, P.; Kukuca, P.; Barta, D.; Labuda, R.; Drozdiel, P.; Pukalskas, S. The issue of balancing internal combustion engines with non-conventional crank mechanism. Commun. Sci. Lett. Univ. Zilina 2017, 19, 36-41.

5. Kukuca, P.; Barta, D.; Labuda, R.; Gechev, T. Engine with unconventional crank mechanism FIK1. In Proceedings of the 3rd International Scientific Conference on Innovative Technologies in Engineering Production (ITEP), Bojnice, Slovakia, 11-13 September 2018; Stancekova, D., Vasko, M., Rudawska, A., Cubonova, N., Sapietova, A., Mrazik, J., Szabelski, J., Tlach, V., Eds.; Édition Diffusion Presse Sciences: London, UK, 2018.

6. Baran, P.; Stastniak, P.; Kukuca, P.; Brezani, M. Investigation of kinematic parameters of two nonconventional piston machines with wobble board. In Proceedings of the 22nd Slovak-Polish Scientific Conference on Machine Modelling and Simulations (MMS), Sklene Teplice, Slovakia, 5-8 September 2017; Vasko, M., Handrik, M., Jakubovicova, L., Kopas, P., Blatnicka, M., Stalmach, O., Sapietova, A., Saga, M., Eds.; Édition Diffusion Presse Sciences: London, UK, 2018.

7. Kukuca, P.; Barta, D.; Dizo, J.; Caban, J. Piston kinematics of combustion engine with unconventional crank mechanism. In Proceedings of the 3rd International Scientific Conference on Innovative Technologies in Engineering Production (ITEP), Bojnice, Slovakia, 11-13 September 2018; Stancekova, D., Vasko, M., Rudawska, A., Cubonova, N., Sapietova, A., Mrazik, J., Szabelski, J., Tlach, V., Eds.; Édition Diffusion Presse Sciences: London, UK, 2018.

8. Doric, J.Z.; Klinar, I.J. Efficiency of a new internal combustion engine concept with variable piston motion. Therm. Sci. 2014, 18, 113-127. [CrossRef]

9. Drapal, L.; Voparil, J. Torsional vibration of unconventional crankshaft in four-cylinder passenger car engine. In Proceedings of the 21st International Scientific Conference on Transport Means (Transport Means), Juodkrante, Lithuania, 20-22 September 2017.

10. Zhang, X.M.; Wang, Y.Q.; Fang, J. Dynamic simulation of crank-connecting rod-piston mechanism of internal combustion engine based on virtual prototype technology. In Proceedings of the International Conference on Electrical Information and Mechatronics (ICEIM), Jiaozuo, China, 23-25 December 2011.

11. Itu, C.; Scutaru, M.L.; Pruncu, C.I.; Muntean, R. Kinematic and Dynamic Response of a Novel Engine Mechanism Design Driven by an Oscillation Arm. Appl. Sci. 2020, 10, 2733. [CrossRef]

12. Petrescu, F.I.; Petrescu, R.V. An Otto engine dynamic model. Indep. J. Manag. Prod. 2016, 7, 38-48. [CrossRef]

13. Kaplan, H. Mathematical modeling and simulation of high-speed cam mechanisms to minimize residual vibrations. Proc. Inst. Mech. Eng. Part C J. Mech. Eng. Sci. 2014, 228, 2402-2415. [CrossRef]

14. Rząsa, M.; Łukasiewicz, E.; Wójtowicz, D. Test of a new low-speed compressed air engine for energy recovery. Energies 2021, 14, 1179. [CrossRef]

15. Tokhi, M.O.; Al-Miskiry, M.; Brisland, M. Real-time control of air motors using a pneumatic H-bridge. Control Eng. Pract. 2001, 9 , 449-457. [CrossRef]

16. Xu, Q.; Cai, M.; Shi, Y. Dynamic heat transfer model for temperature drop analysis and heat exchange system design of the air-powered engine system. Energy 2014, 68, 877-885. [CrossRef]

17. Fang, Y.; Lu, Y.; Yu, X.; Roskilly, A.P. Experimental study of a pneumatic engine with heat supply to improve the overall performance. Appl. Therm. Eng. 2018, 137, 78-85. [CrossRef]

18. Uszynski, S.; Ambroziak, L.; Kondratiuk, M.; Kulesza, Z. Air consumption analysis in compressed air powered vehicles. In Proceedings of the 23rd International Conference on Methods and Models in Automation and Robotics (MMAR), Miedzyzdroje, Poland, 27-30 August 2018.

19. Fang, Y.; Lu, Y.; Roskilly, A.P.; Yu, X. A review of compressed air energy systems in vehicle transport. Energy Strategy Rev. 2021, 33, 100583. [CrossRef]

20. Yu, Q.H.; Tan, X.; Meng, J.G.; Shang, F.; Hao, X.O. Performance characteristics research on power system of a four-valve compressed air engine. Stroj. Vestn. J. Mech. Eng. 2018, 64, 632-642.

21. Huang, C.Y.; Hu, C.K.; Yu, C.J.; Sung, C.K. Experimental investigation on the performance of a compressed-air driven piston engine. Energies 2013, 6, 1731-1745. [CrossRef]

22. Wang, Y.W.; You, J.J.; Sung, C.K.; Huang, C.Y. The applications of piston type compressed air engines on motor vehicles. In Proceedings of the 37th National Conference on Theoretical and Applied Mechanics (37th-NCTAM)/1st International Conference on Mechanics (1st-ICM), Hsinchu, Taiwan, 8-9 November 2013; Huang, C.Y., Tsai, H.Y., Chang, J.Y., Yeh, M.K., Lin, C.A., Eds.; Elsevier: Amsterdam, The Netherlands, 2014.

23. Fang, Y.; Lu, Y.; Yu, X.; Su, L.; Fan, Z.; Huang, R.; Roskilly, A.P. Study of a hybrid pneumatic-combustion engine under steady-state and transient conditions for transport application. Int. J. Engine Res. 2021, 528-539. [CrossRef]

24. Shi, Y.; Li, F.; Cai, M.; Yu, Q. Literature review: Present state and future trends of air-powered vehicles. J. Renew. Sustain. Energy 2016, 8, 025704. [CrossRef]

25. A Pneumobil: An Air Car. (In Czech). Available online: https://www.abicko.cz/clanek/precti-si-technika-auta/22199/ pneumobil-auto-na-vzduch.html (accessed on 22 May 2021).

26. A Pneumobil from Brno Has Won a Race in Hungary. It Is Possible to Go Even Fifty on the Compressed Air. (In Czech). Available online: https: / / ct24.ceskatelevize.cz/regiony/2486113-pneumobil-z-brna-vyhral-zavody-v-madarsku-na-stlaceny-vzduchse-da-jet-az-padesatkou (accessed on 23 May 2021).

27. The First Car with a Pneumatic Engine in Slovakia. (In Slovak). Available online: https://www.sjf.tuke.sk/kr/katedra/aktuality/ pneumobil (accessed on 21 May 2021). 
28. Zhai, X.; Yu, X.L.; Cai, J.L.; Shen, Y.M. Experimental study on performances of compressed-air engine. Zhejiang Daxue Xuebao (Gongxue Ban). J. Zhejiang Univ. Eng. Sci. 2006, 4, 135-138.

29. Coldea, C. Technical University of Cluj-Napoca student's involvement in international competitions. Ing. Automob. 2011, 18, 26.

30. Pneumobiles of the Brno University of Technology Have Defeated European Elite (In Czech). Available online: https:// www.novinky.cz/veda-skoly/clanek/pneumobily-studentu-brnenske-techniky-porazily-evropskou-elitu-14762 (accessed on 22 May 2021).

31. Chen, P.T.; Nghia, D.D.; Yang, C.J.; Huang, K.D. Study of system integral energy efficiency of a hybrid pneumatic power system. Appl. Sci. 2019, 9, 2333. [CrossRef]

32. BharatAutos. Report: Tata Nano Could Spawn Electric, Hybrid and Air-Powered Variants. Available online: https:/ /bharathautos com/tata-nano-petrol-diesel-electric-hybrid-air-power-cars.html (accessed on 29 July 2021).

33. European Publication Server. Available online: https: / / data.epo.org/publication-server / document?iDocId=4033778\&iFormat=0 (accessed on 2 August 2021).

34. Creutzig, F.; Papson, A.; Schipper, L.; Kammen, D.M. Economic and environmental evaluation of compressed-air cars. Environ. Res. Lett. 2009, 4, 044011. [CrossRef]

35. Dobrodenka, P.; Dobrodenka, A.; Dobrodenka, M.; Gerlici, J.; Lack, T.; Blatnický, M.; Dižo, J.; Harušinec, J. Kinematic Arrangement of the Mechanism with Rotating Cylinders. Patent SK 8035 Y1, 5 March 2018.

36. Gerlici, J.; Sakhno, V.; Yefymenko, A.; Verbitskii, V.; Kravchenko, A.; Kravchenko, K. The stability analysis of two-wheeled vehicle model. In Proceedings of the 22nd Slovak-Polish Scientific Conference on Machine Modelling and Simulations (MMS), Sklene Teplice, Slovakia, 5-8 September 2017; Vasko, M., Handrik, M., Jakubovicova, L., Kopas, P., Blatnicka, M., Stalmach, O., Sapietova, A., Saga, M., Eds.; Édition Diffusion Presse Sciences: London, UK, 2018.

37. Fomin, O.; Gerlici, J.; Lovskaya, A.; Kravchenko, K.; Burlutski, O.; Hauser, V. Peculiarities of the mathematical modelling of dynamic loading on containers in flat wagons transportation. In Proceedings of the 23rd Polish-Slovak Scientific Conference on Machine Modelling and Simulations (MMS), Rydzyna, Slovakia, 4-7 September 2018; Malujda, I., Dudziak, K., Krawiec, P., Talaska, K., Wilczynski, D., Berdychowski, M., Gorecki, J., Wargula, L., Wojtkowiak, D., Eds.; EDP Sciences: Youlis, France, 2019.

38. Lack, T.; Gerlici, J. The assessment of the integration methods for the rail vehicle ride dynamics solution. In Proceedings of the 22nd Slovak-Polish Scientific Conference on Machine Modelling and Simulations (MMS), Sklene Teplice, Slovakia, 5-8 September 2017; Vasko, M., Handrik, M., Jakubovicova, L., Kopas, P., Blatnicka, M., Stalmach, O., Sapietova, A., Saga, M., Eds.; Édition Diffusion Presse Sciences: London, UK, 2018.

39. Loveikin, V.S.; Pochka, K.I.; Romasevich, Y.O.; Pochka, O.B. Dynamic analysis of roller forming installation about a crank connecting rod the driving mechanism. Opir Mater. I Teor. Sporud-Strength Mater. Theory Struct. 2019, 102, 91-108. [CrossRef]

40. Krawiec, P. Analysis of selected dynamic features of a two-wheeled transmission system. J. Theor. Appl. Mech. 2017, 55, 461-467. [CrossRef]

41. Kujawski, M.; Krawiec, P. Analysis of generation capabilities of noncircular cogbelt pulleys on the example of a gear with an elliptical pitch line. J. Manuf. Sci. Eng. Trans. ASME 2011, 133, 051006. [CrossRef]

42. Stoffels, H. Balancing driveability, NVH, and fuel consumption on automotive powertrains using integrated simulation techniques. Proc. Inst. Mech. Eng. Part K J. Multi Body Dyn. 2017, 231, 556-567. [CrossRef]

43. Hauser, V.; Nozhenko, O.; Kravchenko, K.; Loulova, M.; Gerlici, J.; Lack, T. Proposal of a steering mechanism for tram bogie with three axle boxes. In Proceedings of the 12th International Scientific Conference of Young Scientists on Sustainable, Modern and Safe Transport, High Tatras, Slovakia, 31 May-2 June 2017; Bujnak, J., Guagliano, M., Eds.; Elsevier: Amsterdam, The Netherlands, 2014.

44. Flizikowski, J.; Macko, M.; Czerniak, J.; Mroziński, A. Implementation of genetic algorithms into development of mechatronic multiedge's grinder design. In Proceedings of the ASME International Mechanical Engineering Congress and Exposition (IMECE), Denver, CO, USA, 11-17 November 2011.

45. Frankovský, P.; Hroncová, D.; Delyová, I.; Hudák, P. Inverse and forward dynamic analysis of two link manipulator. Procedia Eng. 2012, 48, 158-163. [CrossRef]

46. Bajla, J.; Bronček, J.; Antala, J.; Sekerešová, D. Engineering Tables. Selection of Standards, 1st ed.; Slovak Office of Standards, Metrology and Testing: Bratislava, Slovak, 2014. (In Slovak)

47. Hlavňa, V.; Kukuča, P.; Isteník, R.; Labuda, R.; Liščák, Š. A Transport Mean-Its Engine, 1st ed.; University of Žilina: Žilina, Slovak, 2007. (In Slovak)

48. Noga, M. Selected issues of the indicating measurements in a spark ignition engine with an additional expansion process. Appl. Sci. 2017, 7, 295. [CrossRef] 